\title{
Actuator Saturation in Individual Blade Control of Rotorcraft
}

\author{
Ashwani K. Padthe, Peretz P. Friedmann† and Dennis S. Bernstein ${ }^{\ddagger}$ \\ Department of Aerospace Engineering \\ The University of Michigan, Ann Arbor, MI, 48109
}

\begin{abstract}
The effect of actuator saturation on the vibration and noise reduction capabilities of actively controlled trailing-edge flaps and microflaps is examined. A new approach to handling actuator saturation in the HHC algorithm is developed based on constrained nonlinear optimization techniques. The performance of this approach in reducing vibrations and noise is compared to three existing approaches at various flight conditions. The results indicate that truncating or scaling the optimal flap/microflap deflection can significantly compromise the vibration or noise reduction performance. By comparison, the auto-weighting approach and the new optimization based approach yield far better performance. However, the optimization approach takes less computational time and performs better in the case of multiple control surfaces as it utilizes all of them to the maximum possible extent.
\end{abstract}

\section{Nomenclature}

$\begin{array}{ll}b & \text { Airfoil semi-chord }=c / 2 \\ c & \text { Airfoil chord } \\ c_{p} & \text { Pressure coefficient on the airfoil surface } \\ C_{T} & \text { Rotor thrust coefficient } \\ \mathrm{C}_{d f} & \text { Parasitic drag coefficient of the fuselage } \\ \mathrm{C}_{W} & \text { Helicopter Weight coefficient } \\ D_{0}, D_{1} & \text { Generalized flap motions } \\ e & \text { Blade root offset from the center of rotation } \\ \mathbf{f} & \text { Generalized load vector } \\ F_{H X 4}, F_{H Y 4}, & \text { Nondimensional } 4 / \text { rev hub shears } \\ F_{H Z 4} & \text { Reduced frequency }=\omega b / U \\ k & \text { Blade length } \\ L_{b} & \text { Spanwise dimension of blade segment with microflap } \\ L_{c} & \text { Mach number } \\ M & \text { Blade mass } \\ M_{b} & \text { Nondimensional 4/rev hub moments } \\ M_{H X 4}, M_{H Y 4}, & \text { Total yawing moment about the hub } \\ M_{H Z 4} & \text { Number of rotor blades } \\ M_{H z 1} & 6^{\text {th }}-17^{\text {th }} \text { harmonic components of BVI noise } \\ N_{b} & \text { Number of lag terms } \\ N_{H 06}, N_{H 07}, & \text { Rotor power } \\ \cdots, N_{H 17} & \text { Nondim. surface pressure distribution } \\ n_{L} & \end{array}$

*Postdoctoral Researcher, Member AIAA.

${ }^{\dagger}$ François-Xavier Bagnoud Professor, Fellow AIAA.

${ }^{\ddagger}$ Professor, Member AIAA. 


\begin{tabular}{|c|c|}
\hline Q & Output weighting \\
\hline $\mathbf{R}$ & Control input weighting \\
\hline$R$ & Blade radius \\
\hline$s$ & Laplace variable \\
\hline $\bar{s}$ & Nondim. Laplace variable $=s b / U$ \\
\hline $\mathrm{T}$ & Sensitivity matrix \\
\hline$t$ & Time \\
\hline $\bar{t}$ & Reduced time $=\frac{1}{b} \int_{0}^{t} U(\tau) d \tau$ \\
\hline$U(t)$ & Freestream velocity, time-dependent \\
\hline $\mathbf{u}$ & Control input vector \\
\hline$v, \Delta v, \Delta v_{a}$ & $\begin{array}{l}\text { Components of the local velocity at any point on the surface of the airfoil due to thickness, } \\
\text { camber, and angle of attack, respectively }\end{array}$ \\
\hline$V$ & Freestream velocity for the airfoil \\
\hline$w$ & Disturbance \\
\hline $\mathrm{w}$ & Relation between the output and the disturbance \\
\hline$W_{\alpha}$ & Relative weighting parameter \\
\hline$X_{A}$ & Offset between the aerodynamic center and the elastic axis \\
\hline$X_{F A}, Z_{F A}$ & Longitudinal and vertical offsets between rotor hub and helicopter aerodynamic center \\
\hline$X_{F C}, Z_{F C}$ & Longitudinal and vertical offsets between rotor hub and helicopter center of gravity \\
\hline$X_{I b}$ & Offset of the blade cross-sectional center of mass from the elastic axis \\
\hline$x_{c}$ & Spanwise location of center of microflap segment \\
\hline $\mathbf{z}$ & Output vector \\
\hline$\alpha$ & Airfoil angle of attack \\
\hline$\alpha_{R}$ & Rotor shaft angle \\
\hline$\beta_{p}$ & Blade precone angle \\
\hline$\delta$ & Flap deflection \\
\hline$\delta_{N C}, \delta_{N S}$ & $\mathrm{~N} /$ rev cosine and sine components of flap deflection \\
\hline$\delta_{\text {limit }}$ & Saturation limit on flap deflection \\
\hline$\gamma$ & Lock number \\
\hline$\gamma_{n}$ & Rational approximant poles \\
\hline$\mu$ & Helicopter advance ratio \\
\hline$\omega$ & Oscillation frequency \\
\hline $\bar{\omega}$ & Nondim. normal velocity distribution \\
\hline$\Omega$ & Rotor angular speed \\
\hline$\omega_{F}, \omega_{L}, \omega_{T}$ & Blade flap, lead-lag and torsional natural frequencies \\
\hline$\psi$ & Azimuth angle \\
\hline$\sigma$ & Rotor solidity \\
\hline$\theta_{\mathrm{tw}}$ & Built-in twist angle \\
\hline
\end{tabular}

\section{Introduction}

Noise and vibrations are a major source of concern in the design and maintenance of helicopters. Vibrations cause crew and passenger discomfort and reduce the airframe and component fatigue lives resulting in high maintenance costs. High levels of blade-vortex interaction (BVI) noise are a primary hindrance to the community acceptance of a civilian helicopter. During the last two decades, various active control approaches, such as the higher harmonic control, ${ }^{1}$ individual blade control (IBC),${ }^{2,3}$ actively controlled conventional plain trailing-edge flaps $(\mathrm{ACF}),{ }^{4-7}$ the active twist rotor (ATR) ${ }^{8,9}$ and the microflaps ${ }^{10-12}$ have been explored and shown to have potential for vibration and noise reduction in rotorcraft. Approaches such as the IBC and the ATR which rely on deflecting or twisting the entire blade require high actuation power. By contrast, the ACFs (depicted in Fig. 1) and the microflaps (depicted in Fig. 2) require significantly less power for actuation. However, the actuation devices used to operate the ACFs have limited torque capacities and hence are subject to amplitude saturation. Similarly for microflaps, the thickness of the airfoil imposes a limitation on the size of the microflap thus constraining the maximum deflection of the microflap. Saturation constraints introduce nonlinearities into a linear system, forcing the control-system to operate in a mode for which it was not designed resulting in a significant degradation of its performance. ${ }^{13}$ Therefore, it is 
important to consider the effects of actuator saturation in the various active control approaches.

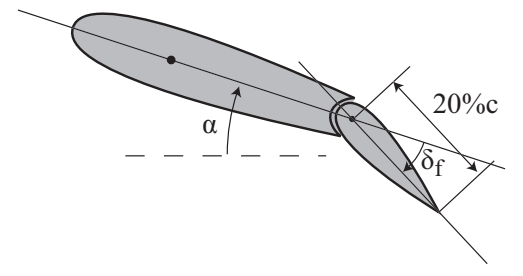

Figure 1. A $20 \%$ c conventional plain flap cross-section.

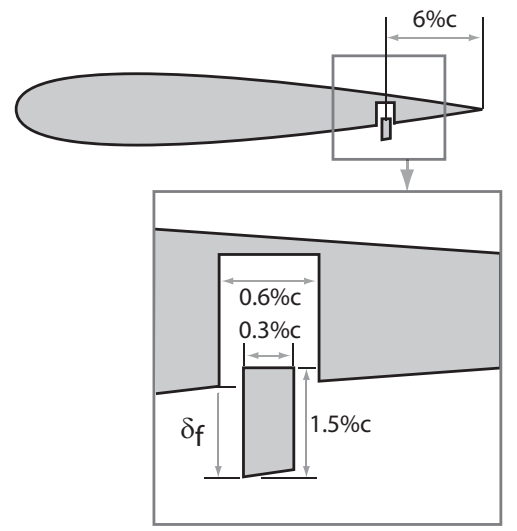

Figure 2. An oscillating microflap cross-section.

The higher harmonic control (HHC) algorithm has been widely employed for closed-loop vibration and noise reduction studies using the conventional trailing-edge flaps ${ }^{4,6,14,15}$ and more recently the microflaps. ${ }^{11}$ These studies have shown that the vibration and noise reduction performance of the flaps (or microflaps) is best when implemented in a dual flap configuration. A good review of the HHC algorithm and its variants was written by Johnson in Ref. 16. A detailed description of the algorithm, including robustness and stability analyses, can be found in Ref. 15. However, the effect of actuator saturation on the performance of the HHC algorithm has received only limited attention. A preliminary investigation of the effect of actuator saturation on the vibration reduction capabilities of the HHC algorithm using a conventional plain flap was considered in Ref. 17. Three different approaches for constraining the flap deflections involving an a posteriori modification of the optimal control input obtained from the HHC algorithm were examined. These approaches were denoted as 1) truncation (TR) - simply clipping the optimal flap deflection whenever it exceeds the saturation limits, 2) scaling (SC) - uniformly scaling down the optimal flap deflection such that it never exceeds the saturation limits, and 3) auto-weighting (AW) - consisting of an iterative adjustment of the control weighting matrix in the HHC algorithm such that flap deflection is properly constrained. The TR and SC approaches were inconsistent and produced only limited vibration reduction whereas the AW approach produced excellent performance resulting in a $90 \%$ reduction of vibration. Despite its effectiveness, the AW approach has shortcomings. It uses an a priori guess of the upper bound on the optimal control weighting, and requires several iterations to converge, thus increasing the computational cost. Furthermore, for multiple flaps (or microflaps), the control weighting on the multiple control surfaces is identical. Thus control input is optimal only for one of the control surfaces leaving the others under-utilized. Using a different control weighting for each of the different control surfaces implies significant increases in computational cost.

The shortcomings of the AW approach can be remedied by a new approach based on constrained nonlinear optimization developed in this paper. In this approach, actuator saturation constraints formulated as inequality constraints on the control surface deflection are combined with the minimization of the quadratic cost function in the HHC algorithm resulting in a constrained nonlinear optimization problem. Unlike the approaches described in Ref. 17, this approach, denoted here as the optimization (OPT) approach, accounts for the presence of saturation nonlinearities in an a priori manner involving direct modifications to the HHC algorithm. A similar approach to handling actuator saturation was proposed in Ref. 18 for vibration reduction using a single trailing-edge flap. However, the performance of this approach that is particularly effective for configurations involving multiple control surfaces was not considered. 
The overall objective of this paper is to examine the OPT approach in detail and compare it to the TR, $\mathrm{SC}$, and AW approaches. The specific objectives are:

1. Develop a computationally efficient approach to handling actuator saturation in the HHC algorithm, that is suitable for multiple control surfaces, without compromising on the controller performance.

2. Compare the computational cost and performance of the various actuator saturation approaches for reducing BVI noise and vibrations on a representative rotor configuration using the single and dual configurations of a $20 \%$ c conventional plain trailing-edge flap and a $1.5 \%$ c microflap.

3. Compare the vibration reduction performance of the various saturation approaches at two different flight conditions: (a) low-speed descending flight where vibrations are high due to blade-vortex interaction and (b) high-speed cruise condition.

\section{Description of the Aeroelastic Analysis Code}

The comprehensive rotorcraft simulation code AVINOR (Active Vibration and Noise Reduction), which has been extensively validated in earlier studies ${ }^{6,11,19,20}$ is used for all the active vibration reduction studies presented in this paper. A brief description of the various components of the code is provided next, for additional details see Ref. 19.

\section{II.A. Aerodynamic model}

The blade/flap and blade/microflap sectional aerodynamic loads for attached flow are calculated using a rational function approximation (RFA) based nonlinear reduced order model constructed from CFD data. ${ }^{10,21}$ This model provides unsteady lift, moment, as well as drag predictions for both plain flap and microflap configurations. The CFD based RFA model is linked to a free wake model, ${ }^{6,20}$ which produces a spanwise and azimuthally varying inflow distribution. For separated flow regime, the aerodynamic loads are calculated using the ONERA dynamic stall model. ${ }^{6}$

\section{II.B. Structural dynamic model}

The structural dynamic model consists of a four-bladed hingeless rotor, having fully coupled flap-lag-torsional dynamics for each blade. The structural dynamic model is geometrically nonlinear, due to moderate blade deflections. The structural equations of motion are discretized using the global Galerkin method, based upon the free vibration modes of the rotating blade. The dynamics of the blade are represented by three flap, two lead-lag, and two torsional modes. Free vibration modes of the blade were obtained using the first nine exact non-rotating modes of a uniform cantilevered beam. The effects of control surfaces such as the trailing-edge plain flap or the microflap on the structural properties of the blade were neglected. Thus, the control surfaces influence the blade behavior only through their effect on the aerodynamic and inertial loads.

\section{II.C. Coupled aeroelastic response/trim solution}

The combined structural and aerodynamic equations are represented by a system of coupled ordinary differential equations with periodic coefficients in state-variable form. The trim employed is a propulsive trim procedure where three force equations (longitudinal, lateral, and vertical) and three moment equations (roll, pitch, and yaw) corresponding to a helicopter in free flight are enforced. A simplified tail rotor model, based on uniform inflow and blade element theory, is used. The six trim variables are the rotor shaft angle $\alpha_{R}$, the collective pitch $\theta_{0}$, the cyclic pitch $\theta_{1 s}$ and $\theta_{1 c}$, the tail rotor constant pitch $\theta_{0 t}$, and lateral roll angle $\phi_{R}$. The coupled trim/aeroelastic equations are solved in time using the ODE solver DDEABM, which is a predictor-corrector based Adams-Bashforth differential system solver.

\section{II.D. Acoustic model}

The acoustic calculations are based on a modified version of the WOPWOP code, where helicopter noise is obtained from the Ffowcs-Williams Hawkings equation with the quadrupole term neglected. ${ }^{22}$ The version of WOPWOP used in the code was modified to account for a fully flexible blade model that is compatible with the structural dynamic model described earlier. In previous studies, ${ }^{6,23}$ the chordwise pressure distribution 
on the surface of the blade, a required input to the acoustic computations, was obtained using an extended RFA approach. The extended RFA approach used frequency domain pressure data obtained from the doublet lattice flow solver, described in detail in Ref. 23. Generating the extended RFA models using CFD based pressure distribution data is computationally expensive. To reduce the cost the blade pressure distributions are obtained from an approximate velocity superposition method. ${ }^{24}$ Based on potential flow theory, the pressure distribution on the surface of the airfoil is related to the local velocity distribution that is assumed to result from three independent contributions:

$$
\mathrm{C}_{p}=\left(\frac{v}{V} \pm \frac{\Delta v}{V} \pm \frac{\Delta v_{a}}{V}\right)^{2}
$$

where the velocity ratios $\frac{v}{V}, \frac{\Delta v}{V}$, and $\frac{\Delta v_{a}}{V}$ are contributions due to airfoil thickness, camber, and angle of attack, respectively. The signs in Eq. 1 are positive for the upper surface and negative for the lower surface of the airfoil. For the symmetric NACA 0012 airfoil used in the study, $\frac{\Delta v}{V}=0$, and the values of the other two components are found from the approach described in Ref. 24. Since this approach is based on the potential flow theory it is not quite compatible with the CFD based RFA model. However, it represents an acceptable approximation because the lift coefficients from which the pressure distributions are obtained, are based on the CFD+RFA model that accounts for compressibility, viscosity, and unsteady effects.

\section{The Higher Harmonic Control Algorithm}

Active control of vibration and noise is implemented using the HHC algorithm used extensively for active control of vibration and noise in rotorcraft. ${ }^{6,15}$ The algorithm is based on the assumption that the helicopter can be represented by a linear model relating the output of interest $\mathbf{z}$ to the control input $\mathbf{u}$. The measurement of the plant output and update of the control input are not performed continuously, but rather at specific times $t_{k}=k \tau$, where $\tau$ is the time interval between updates during which the plant output reaches a steady state. In actual implementation of the algorithm, this time interval may be one or more revolutions. A schematic of the HHC architecture as implemented on a helicopter is shown in Fig. 3. The

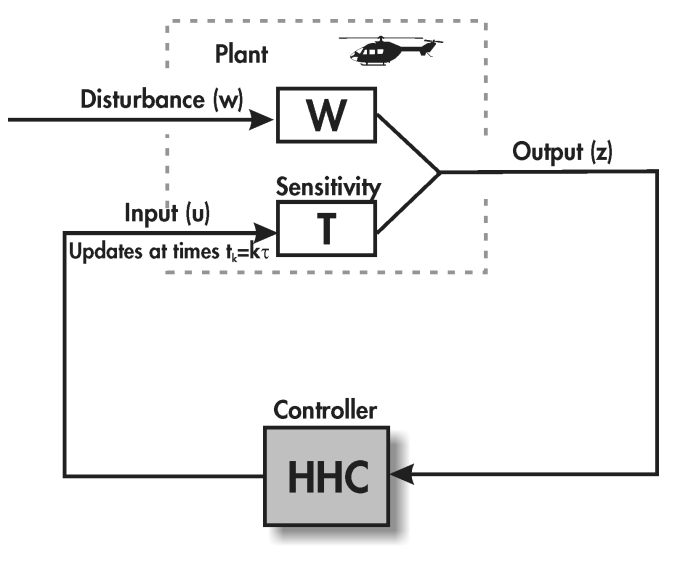

Figure 3. Higher harmonic control architecture

disturbance $\mathbf{w}$ is representative of the helicopter operating condition. The output vector at the $k^{t h}$ time step is given by

$$
\mathbf{z}_{k}=\mathbf{T} \mathbf{u}_{k}+\mathbf{W} \mathbf{w}
$$

where the sensitivity $\mathbf{T}$ is given by

$$
\mathbf{T}=\frac{\partial \mathbf{z}}{\partial \mathbf{u}}
$$

At the initial condition, $k=0$,

$$
\mathbf{z}_{0}=\mathbf{T} \mathbf{u}_{0}+\mathbf{W} \mathbf{w} .
$$

Subtracting Eq. (4) from Eq. (2) to eliminate the unknown w yields

$$
\mathbf{z}_{k}=\mathbf{z}_{0}+\mathbf{T}\left(\mathbf{u}_{k}-\mathbf{u}_{0}\right) .
$$


The controller is based on the minimization of a general quadratic cost function

$$
J\left(\mathbf{z}_{k}, \mathbf{u}_{k}\right)=\mathbf{z}_{k}^{\mathrm{T}} \mathbf{Q} \mathbf{z}_{k}+2 \mathbf{z}_{k}^{\mathrm{T}} \mathbf{S} \mathbf{u}_{k}+\mathbf{u}_{k}^{\mathrm{T}} \mathbf{R} \mathbf{u}_{k} .
$$

However, in most applications, the cross-weighting term in Eq. (6) is neglected and the cost function simplifies to

$$
J\left(\mathbf{z}_{k}, \mathbf{u}_{k}\right)=\mathbf{z}_{k}^{\mathrm{T}} \mathbf{Q} \mathbf{z}_{k}+\mathbf{u}_{k}^{\mathrm{T}} \mathbf{R} \mathbf{u}_{k}
$$

The optimal control input is determined from the requirement

$$
\frac{\partial J\left(\mathbf{z}_{k}, \mathbf{u}_{k}\right)}{\partial \mathbf{u}_{k}}=0
$$

which yields the optimal control law $\mathbf{u}_{k, \text { opt }}$, given by

$$
\mathbf{u}_{k, \text { opt }}=-\left(\mathbf{T}^{\mathrm{T}} \mathbf{Q} \mathbf{T}+\mathbf{R}\right)^{-1}\left(\mathbf{T}^{\mathrm{T}} \mathbf{Q}\right)\left(\mathbf{z}_{0}-\mathbf{T} \mathbf{u}_{0}\right) .
$$

Combining Eqs. (5), (7) and (9), the minimum cost is found to be

$$
J\left(\mathbf{z}_{k}, \mathbf{u}_{k, \text { opt }}\right)=\left(\mathbf{z}_{0}-\mathbf{T} \mathbf{u}_{0}\right)^{\mathrm{T}}\left[\mathbf{Q}-(\mathbf{Q T}) \mathbf{D}^{-1}\left(\mathbf{T}^{\mathrm{T}} \mathbf{Q}\right)\right]\left(\mathbf{z}_{0}-\mathbf{T} \mathbf{u}_{0}\right) .
$$

where

$$
\mathbf{D}=\mathbf{T}^{\mathrm{T}} \mathbf{Q} \mathbf{T}+\mathbf{R}
$$

This is a classical version of the HHC algorithm that yields an explicit relation for the optimal control input. Another version of the HHC algorithm where the sensitivity matrix $\mathbf{T}$ is updated using least-squares methods after every control update is known as the adaptive or recursive HHC and is discussed in Ref. 15.

For a 4 -bladed rotor, the control input $\mathbf{u}_{k}$ is a combination of $2 / \mathrm{rev}, 3 / \mathrm{rev}, 4 / \mathrm{rev}$, and $5 / \mathrm{rev}$ harmonic amplitudes of the control surface deflection:

$$
\mathbf{u}_{k}=\left[\delta_{2 c}, \delta_{2 s}, \ldots, \delta_{5 c}, \delta_{5 s}\right]^{T} .
$$

The term 'control surface' refers to both the microflap and the conventional plain trailing-edge flap. The total control surface deflection is given by

$$
\delta\left(\psi, \mathbf{u}_{k}\right)=\sum_{N=2}^{5}\left[\delta_{N c} \cos (N \psi)+\delta_{N s} \sin (N \psi)\right] .
$$

where the quantities $\delta_{N c}$ and $\delta_{N s}$ correspond to the cosine and sine components of the $\mathrm{N} /$ rev control input harmonic. When multiple control surfaces are used, the control surface deflections are given by

$$
\delta_{i}\left(\psi, \mathbf{u}_{k}\right)=\sum_{N=2}^{5}\left[\delta_{N c i} \cos (N \psi)+\delta_{N s i} \sin (N \psi)\right],
$$

where $i=1, \ldots, N_{\delta}$ and $N_{\delta}$ is the total number of control surfaces. The control vector $\mathbf{u}_{k}$ is then given by

$$
\mathbf{u}_{k}=\left[\delta_{2 c 1}, \delta_{2 s 1}, \ldots, \delta_{5 c 1}, \delta_{5 s 1}, \ldots, \delta_{2 c N_{\delta}}, \delta_{2 s N_{\delta}}, \ldots, \delta_{5 c N_{\delta}}, \delta_{5 s N_{\delta}}\right]^{T} .
$$

For vibration reduction (VR) studies, the output vector $\mathbf{z}_{k}$ consists of $4 /$ rev vibratory hub shears and moments:

$$
\mathbf{z}_{\mathrm{VR}}=\left[\begin{array}{c}
F_{H X 4} \\
F_{H Y 4} \\
F_{H Z 4} \\
M_{H X 4} \\
M_{H Y 4} \\
M_{H Z 4}
\end{array}\right]
$$


The weighting matrix $\mathbf{Q}$ in the cost function (see Eq. 7) is a diagonal matrix, and, for vibration control, is described by six weights corresponding to the three vibratory hub shears and the three vibratory hub moments. Based on previous studies, ${ }^{6,11}$ the weights for the hub shears were assumed to be identical, and a similar assumption was used for the weights of the hub moments. The weighting matrix used in this study for vibration reduction, $\mathbf{Q}_{\mathrm{VR}}$, is:

$$
\mathbf{Q}_{\mathrm{VR}}=\left[\begin{array}{cccccc}
1 & 0 & 0 & 0 & 0 & 0 \\
0 & 1 & 0 & 0 & 0 & 0 \\
0 & 0 & 1 & 0 & 0 & 0 \\
0 & 0 & 0 & 10 & 0 & 0 \\
0 & 0 & 0 & 0 & 10 & 0 \\
0 & 0 & 0 & 0 & 0 & 10
\end{array}\right]
$$

For BVI noise reduction (NR) studies, the output vector consists of the $6^{\text {th }}-17^{\text {th }}$ blade passage frequency harmonic components of BVI noise (the most significant part of BVI noise), as measured by a microphone installed at a suitable location. This location is usually on the skid or landing gear of the helicopter, and

$$
\mathbf{z}_{\mathrm{NR}}=\left[\begin{array}{c}
N_{H 06} \\
N_{H 07} \\
N_{H 08} \\
\vdots \\
N_{H 17}
\end{array}\right]
$$

The noise control law is identical to the control law used for vibration reduction. The weighting matrix used in this study for NR is:

$$
\mathbf{Q}_{\mathrm{NR}}=\left[\begin{array}{ccccccc}
1 & 0 & 0 & 0 & \ldots & \ldots & 0 \\
0 & 1 & 0 & 0 & \ldots & \ldots & 0 \\
0 & 0 & 1 & 0 & \ldots & \ldots & 0 \\
& & & \ddots & & & \\
0 & \ldots & \ldots & 0 & 1 & 0 & 0 \\
0 & \ldots & \ldots & 0 & 0 & 1 & 0 \\
0 & \ldots & \ldots & 0 & 0 & 0 & 1
\end{array}\right] .
$$

Note that all the components of the BVI noise are weighted equally.

For simultaneous vibration and noise reduction (SR) problems, a combined output vector is defined by

$$
\mathbf{z}_{\mathrm{SR}}=\left[\frac{\mathbf{z}_{\mathrm{VR}}}{\mathbf{z}_{\mathrm{NR}}}\right],
$$

where the vector $\mathbf{z}_{\mathrm{SR}}$ is simply a partitioned combination of the vibration and noise levels. The combined weighting matrix $\mathbf{Q}_{\mathrm{SR}}$ is defined as

$$
\mathbf{Q}_{\mathrm{SR}}=\left[\begin{array}{cc}
\left(W_{\alpha}\right) \cdot\left[\mathbf{Q}_{\mathrm{VR}}\right] & 0 \\
0 & \left(1-W_{\alpha}\right) \cdot\left[\mathbf{Q}_{\mathrm{NR}}\right]
\end{array}\right],
$$

where $W_{\alpha}$ is a scalar factor used to adjust the relative weighting between noise and vibration as objectives for the controller. When $W_{\alpha}=1$, the control effort is focused on vibration reduction, and when $W_{\alpha}=0$, only noise is reduced by the controller. During approach to landing, BVI noise is the main priority, while vibration is the goal at cruise conditions. Therefore, in an actual implementation the weighting factor can be adjusted by the controller depending on the desired outcome. The weighting matrices $\mathbf{Q}_{\mathrm{VR}}$ and $\mathbf{Q}_{\mathrm{NR}}$ used 
for simultaneous noise and vibration reduction performed in this study are:

$$
\mathbf{Q}_{\mathrm{VR}}=\left[\begin{array}{cccccc}
1 & 0 & 0 & 0 & 0 & 0 \\
0 & 1 & 0 & 0 & 0 & 0 \\
0 & 0 & 10 & 0 & 0 & 0 \\
0 & 0 & 0 & 10 & 0 & 0 \\
0 & 0 & 0 & 0 & 10 & 0 \\
0 & 0 & 0 & 0 & 0 & 10
\end{array}\right],
$$

and

$$
\mathbf{Q}_{\mathrm{NR}}=\left[\begin{array}{ccccccc}
100 & 0 & 0 & 0 & \ldots & \ldots & 0 \\
0 & 100 & 0 & 0 & \ldots & \ldots & 0 \\
0 & 0 & 100 & 0 & \ldots & \ldots & 0 \\
& & & \ddots & & & \\
0 & \ldots & \ldots & 0 & 100 & 0 & 0 \\
0 & \ldots & \ldots & 0 & 0 & 100 & 0 \\
0 & \ldots & \ldots & 0 & 0 & 0 & 100
\end{array}\right] .
$$

\section{Actuator Saturation}

Most actuation devices used for on blade control of rotorcraft vibrations and noise are subject to amplitude saturation. Furthermore the actuation amplitudes have to be limited so as to avoid undesirable interactions between the primary flight control system and the on blade controller. For a microflap the maximum deflection is constrained by its size, usually $1.5 \%$ of the chord. For a conventional trailing-edge flap the maximum deflection is set to $4^{\circ}$. To study the effects of saturation on the controller performance, four different approaches to implementing actuator saturation in the $\mathrm{HHC}$ algorithm are considered: these are truncation, scaling, auto-weighting, and optimization. In the truncation approach, the unconstrained optimal control input is clipped whenever it exceeds the limiting amplitude, thus the control surface deflection is

$$
\delta\left(\psi, \mathbf{u}_{k}\right)=\left\{\begin{array}{cl}
\delta\left(\psi, \mathbf{u}_{k}\right), & \left|\delta\left(\psi, \mathbf{u}_{k}\right)\right|<\delta_{\text {limit }} \\
\operatorname{sgn}\left(\delta\left(\psi, \mathbf{u}_{k}\right)\right) \cdot \delta_{\text {limit }}, & \left|\delta\left(\psi, \mathbf{u}_{k}\right)\right| \geq \delta_{\text {limit }}
\end{array}\right.
$$

where $\delta_{\text {limit }}$ is the saturation limit on the control surface deflection.

In the scaling approach, the control input is given by

$$
\delta\left(\psi, \mathbf{u}_{k}\right)=\frac{\delta_{\text {limit }}}{\max \left(\left|\delta_{\mathrm{opt}}\left(\psi, \mathbf{u}_{k}\right)\right|\right)} \cdot \delta_{\mathrm{opt}}\left(\psi, \mathbf{u}_{k}\right),
$$

where $\delta_{\text {opt }}\left(\psi, \mathbf{u}_{k}\right)$ is the optimal control input obtained using the HHC algorithm without the saturation constraints. Each harmonic component of the optimal control surface deflection is scaled by a common factor such that the maximum deflection is equal to the saturation limit.

In the third approach denoted as auto-weighting the control weighting matrix, $\mathbf{R}$ in Eq. (7), is updated so as to restrict the control surface deflection. The control weighting matrix $\mathbf{R}$ penalizes the control input and thus can be used to constrain the maximum control surface deflection. However, the value of $\mathbf{R}$ required to constrain the control input amplitude within the saturation limits is not known a priori. Hence, an iterative approach which adjusts the value of $\mathbf{R}$ is used. The weighting matrix $\mathbf{R}$ is represented as:

$$
\mathbf{R}=c_{w u} \mathbf{I}
$$

where $c_{w u}$ is a scalar and $\mathbf{I}$ is the identity matrix. In this approach all harmonic components of the control input vector are weighted equally. If the control surface deflection is overconstrained, the controller reduces the value of $c_{w u}$. If the control surface deflection is underconstrained, the controller increases the value of $c_{w u}$. A new optimal control is calculated using the updated value of $c_{w u}$, obtained as follows:

1. Set $c_{w u}^{-}=0$ and $c_{w u}^{+}=c_{\max }$. 
2. Set $c_{w u}=\frac{1}{2}\left(c_{w u}^{-}+c_{w u}^{+}\right)$

3. Calculate a new optimal control input.

If the flap deflection is properly constrained $\left(\left|\delta_{\max }\right|=\delta_{\text {limit }} \pm 5 \%\right)$, end the algorithm.

If the flap deflection is underconstrained $\left(\left|\delta_{\max }\right|>\delta_{\text {limit }}\right)$, set $c_{w u}^{-}=\frac{1}{2}\left(c_{w u}^{-}+c_{w u}^{+}\right)$. Go to step 2 .

If the flap deflection is overconstrained $\left(\left|\delta_{\max }\right|<\delta_{\text {limit }}\right)$, set $c_{w u}^{+}=\frac{1}{2}\left(c_{w u}^{-}+c_{w u}^{+}\right)$. Go to step 2 .

This iterative procedure increases or decreases $c_{w u}$ until the optimal control surface deflection converges to the desired deflection limits within a prescribed tolerance. The value of $c_{\max }$ has to be estimated initially and it has to be greater than or equal to the optimum value of $c_{w u}$ that properly constrains the control input. Choosing a very large value for $c_{\max }$ is not recommended since depending on the proximity of $c_{\max }$ to the optimum $c_{w u}$, the AW approach can take several iterations causing an increase in the computational costs. Furthermore, for the case of multiple control surfaces, the number of iterations required for all of them to be properly constrained can be quite high rendering the AW approach impractical. To avoid this situation the same value of $c_{w u}$ is used for all the control surfaces.

A new approach for dealing with actuator saturation in the HHC algorithm is developed in this study. This approach, based on constrained nonlinear optimization techniques, overcomes the limitations associated with the previous approaches. Recall that the HHC algorithm is based on the minimization of a quadratic cost function, given by Eq. (7). The saturation limits can be combined with the minimization of the cost function to yield a constrained optimization problem:

$$
\begin{array}{cl}
\underset{\mathbf{u}_{k}}{\operatorname{minimize}} & J\left(\mathbf{z}_{k}, \mathbf{u}_{k}\right)=\mathbf{z}_{k}^{\mathrm{T}} \mathbf{Q} \mathbf{z}_{k}+\mathbf{u}_{k}^{\mathrm{T}} \mathbf{R} \mathbf{u}_{k}, \\
\text { subject to } & \left|\delta_{i}\left(\psi, \mathbf{u}_{k}\right)\right| \leq \delta_{\text {limit }}, \quad i=1, \ldots, N_{\delta}
\end{array}
$$

where $N_{\delta}$ is the total number of control surfaces. The optimization problem given by Eqs. (28) and (29) is a nonlinear constrained optimization problem with a quadratic objective function and nonlinear inequality constraints, and represents a Nonlinear Programming (NP) problem. Unlike the approaches described earlier, this saturation approach involves direct modifications to the HHC algorithm to account for the presence of saturation in an a priori manner. The resulting optimal control input always satisfies the saturation limits irrespective of the values of $\mathbf{R}$ and $\mathbf{Q}$.

A NP method, Sequential Quadratic Programming (SQP), ${ }^{25,26}$ available in the FMINCON tool in MATLAB, is used to solve the optimization problem given by Eqs. (28) and (29). The SQP method solves a quadratic programming subproblem based on a quadratic approximation of the Lagrangian function. A stand-alone application (a .exe file) capable of performing the optimization is generated using the $m c c-m$ command in Matlab. Subsequently, this application is invoked from the AVINOR code, written in FORTRAN, in order to evaluate the optimum $\mathbf{u}_{k}$. The stand-alone application requires approximately 1 sec to run on a $2.53 \mathrm{GHz}$ Intel Xeon processor in the case of a single control surface. Note that the nonlinear constraints described in Eq. (29) have to be satisfied for all values of the azimuth angle $\psi \in\left[0^{\circ} 360^{\circ}\right]$. In actual numerical implementation, the nonlinear constraints are evaluated and enforced at every integer value of $\psi$ over the range $\left[\begin{array}{ll}0^{\circ} & 360^{\circ}\end{array}\right]$.

\section{Results and Discussion}

The results presented in this section are obtained for a helicopter configuration resembling a four-bladed MBB BO-105 hingeless rotor. The rotor parameters are listed in Table 1. All the values in the table (except $C_{W}, \gamma$, and $\sigma$ ) have been non-dimensionalized using $M_{b}, L_{b}$, and $1 / \Omega$ for mass, length and time, respectively. The mass and stiffness distributions are assumed to be constant along the span of the blade.

The vibratory hub shears and moments are obtained from the integration of the distributed inertial and aerodynamic loads over the entire blade span in the rotating frame. Subsequently, the loads are transformed to the hub-fixed non-rotating system, and the contributions from the individual blades are combined. In this process, the blades are assumed to be identical. Reduction is performed on the $N_{b} /$ rev components, which are the dominant components, of the hub shears and moments.

For noise reduction studies, the acoustic environment in the vicinity of the helicopter is characterized by the noise decibel levels computed on a carpet plane located 1.15R beneath the rotor, as depicted in Fig. 4 . Noise measured by a microphone placed on the right landing skid at the rear is used as the feedback signal 
to the controller. The sharp trailing edge configuration, shown in Fig. 2, was chosen for the microflap. The microflap, $1.5 \% \mathrm{c}$ in height, slides in and out of a cavity, located at $6 \% \mathrm{c}$ from the sharp trailing edge of the airfoil. Two different configurations of microflaps on the rotor blade are considered in this study. The first

Table 1. Rotor parameters used for the computations.

\begin{tabular}{ll}
\hline \hline Dimensional Rotor Data & \\
$R=4.91 \mathrm{~m}$ & \\
$M_{b}=27.35 \mathrm{~kg}$ & \\
$\Omega=425 \mathrm{RPM}$ & \\
Nondimensional Main Rotor Data & \\
$N_{b}=4$ & $e=0.05498 R$ \\
$L_{b}=1.0$ & $\beta_{p}=2.5^{\circ}$ \\
$X_{A}=0$ & $X_{I I b}=0$ \\
$X_{I b}=0$ & $I_{M B 3}=0.0004$ \\
$I_{M B 2}=0$ & $E I_{\zeta \zeta}=0.0105$ \\
$E I_{\eta \eta}=0.0302$ & $\omega_{F 1}=1.124$ \\
$G J_{b}=0.0015$ & $\omega_{F 3}=7.606$ \\
$\omega_{F 2}=3.404$ & $\omega_{L 2}=4.458$ \\
$\omega_{L 1}=0.732$ & $\omega_{T 2}=9.079$ \\
$\omega_{T 1}=3.170$ & $\sigma=0.07$ \\
$\gamma=5.5$ & \\
$\theta_{t w}=-8^{\circ}$ & \\
$\underline{\text { Nondimensional Tail Rotor Data }}$ & \\
$X_{t}=1.20$ & $Z_{t}=0$ \\
Helicopter Data & \\
\hline$C_{W}=0.005$ & $f C_{d f}=0.031$ \\
$X_{F A}=0.0$ & $Z_{F A}=0.3$ \\
$X_{F C}=0.0$ & $Z_{F C}=0.3$ \\
\hline \hline
\end{tabular}

configuration, shown in Figure 5(a), has a single microflap with 0.12R spanwise length centered at 0.75R. The second configuration, shown in Figure 5(b), has two microflaps each with 0.06R spanwise length centered at $0.72 \mathrm{R}$ and $0.92 \mathrm{R}$, respectively. Active control studies were also conducted using a $20 \%$ c conventional plain flap, shown in Fig. 1. The spanwise flap configurations considered are shown in Fig. 6.

\section{V.A. Low-speed results}

Various saturation approaches described earlier are compared in terms of their vibration, noise, and also simultaneous vibration and noise reduction capabilities at a heavy BVI descending flight condition with advance ratio $\mu=0.15$ and descent angle $\alpha_{D}=6.5^{\circ}$. Vibration reduction results are presented first. The non-dimensional 4/rev vibratory hub shears and moments obtained during active vibration reduction using the four different saturation approaches for the single plain flap configuration are compared to the baseline (no active control) vibration levels in Fig. 7. The TR approach yields only a $5 \%$ reduction in the vibration objective while causing some of the vibratory hub loads to increase from the baseline levels. The SC approach yields a $38 \%$ reduction in the vibration objective. The AW and OPT approaches yield similar performance yielding $76 \%$ and $78 \%$ reduction in the vibration objective, respectively. However, the OPT approach takes only 10 control updates (80 rotor revolutions) to converge compared to over 100 control updates ( 800 rotor revolutions) taken by the AW approach. The flap deflection histories over one complete rotor revolution corresponding to the various saturation approaches are shown in Fig. 8. The flap deflection histories corresponding to the AW and OPT approaches show similarities in the azimuthal locations of the peaks and troughs. However, the maximum flap deflection obtained using the OPT approach is closer to 

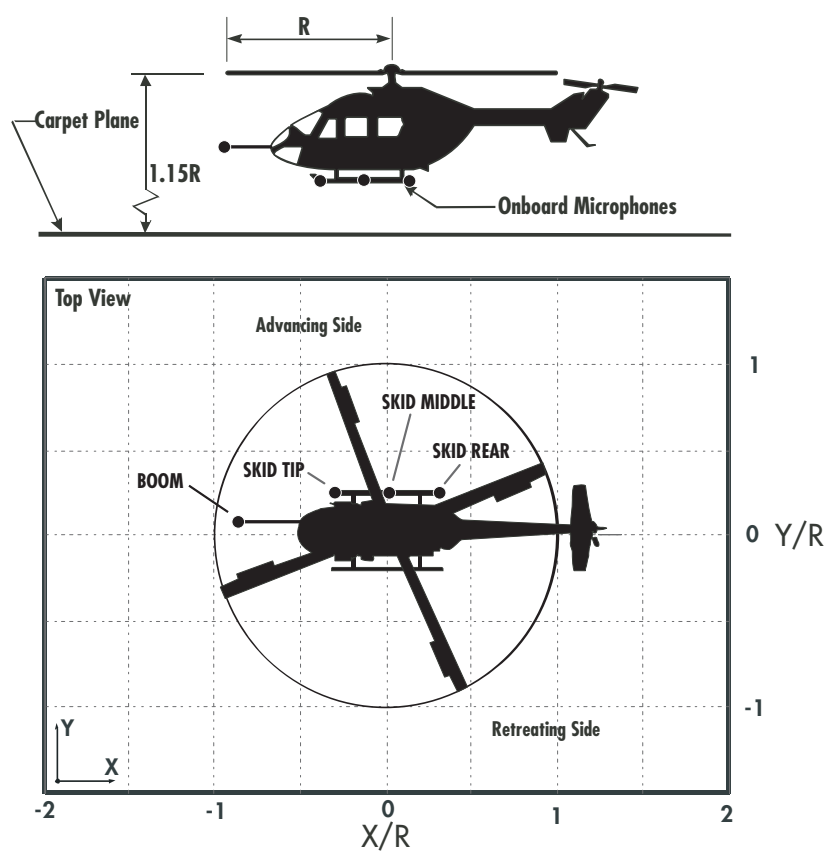

Figure 4. Microphone locations on and around the helicopter for noise measurements.

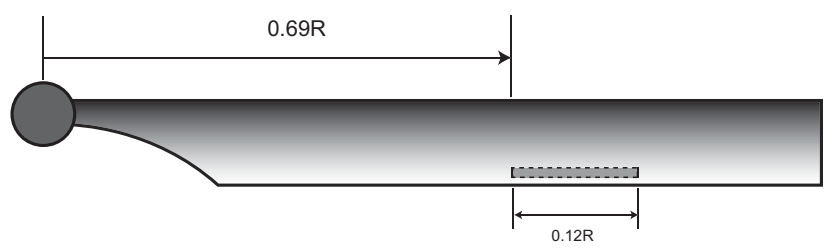

(a) Single Microflap

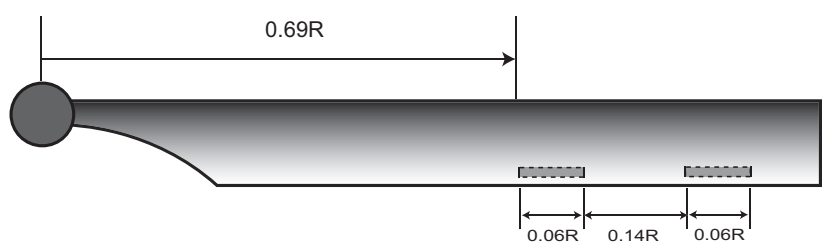

(b) Dual Microflap

Figure 5. Single and dual spanwise configurations of the microflap on the rotor blade

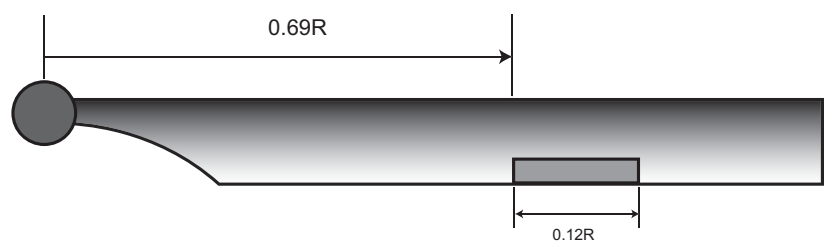

(a) Single plain flap

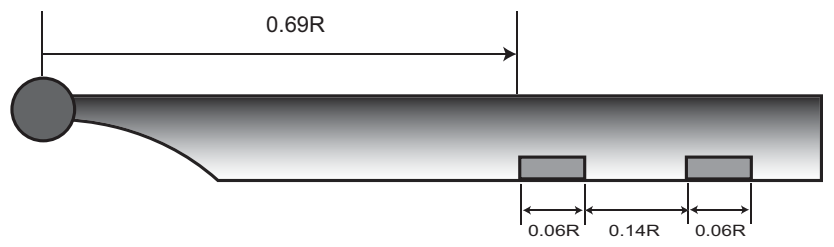

(b) Dual plain flap

Figure 6. Single and dual spanwise configurations of the $20 \%$ c plain flap on the rotor blade 
the saturation limit thus utilizing the flap to the maximum possible degree. This results from the fact that a small tolerance, typically of the order of $10^{-6}$, is used on the constraint violation in the OPT approach.

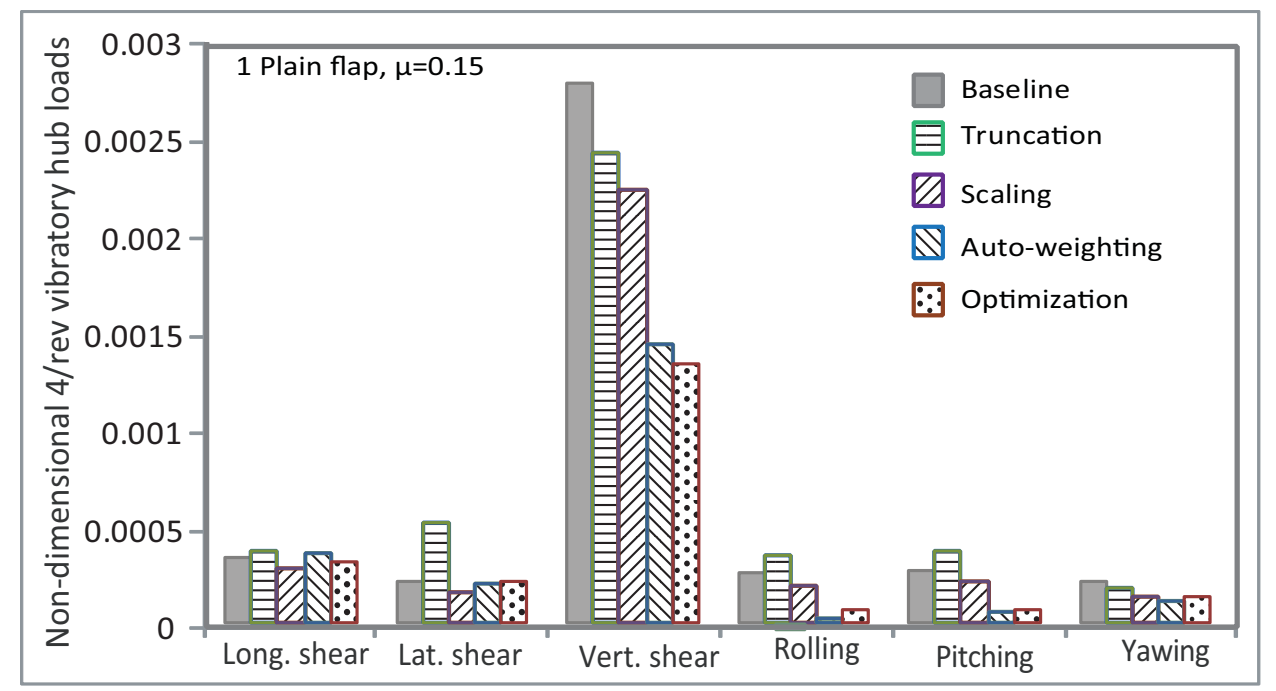

Figure 7. Reduction in 4 /rev vibratory hub shears and moments obtained using the various saturation approaches for the single plain flap configuration at a heavy BVI descending flight condition with $\mu=0.15$.

The 4/rev vibratory hub loads obtained during active vibration reduction using the dual plain flap configuration are compared to the baseline levels in Fig. 9. The TR and SC approaches yield $81 \%$ and $57 \%$ reduction in the vibration objective, respectively. The AW approach yields $95 \%$ reduction whereas the OPT approach yields the best performance with a $98 \%$ reduction in the vibration objective. Significantly better performance obtained using the OPT approach is apparent in the vertical shear component. The inboard and outboard flap deflection histories corresponding to the various saturation approaches are shown in Fig. 10. In the AW approach, the maximum deflection of the inboard flap is significantly less than the saturation limit. This under-utilization of one of the flaps is primarily a result of the fact that the same control weighting $\left(c_{w u}\right.$ in Eq. (27)) is used for both the flaps. Using a different control weighting for the two microflaps results in a significant increase in the computational time. By contrast, the two flaps are optimized individually in the OPT approach as is evident from the constraint inequalities in Eq. (29). Thus, the maximum deflection of both the flaps is equal to the saturation limit within the tolerance limits. This feature of the OPT approach facilitates the use of both the flaps to the maximum possible extent resulting in a better vibration reduction performance.

Similar comparisons are also performed for the single and dual microflap configurations. The 4/rev vibratory hub loads obtained using the various saturation approaches for a single microflap configuration are compared to the baseline levels in Fig. 11. The TR approach yields a $64 \%$ reduction in the vibration objective whereas the SC approach yields no significant reduction. The AW and OPT approaches yield similar performance with $71 \%$ and $70 \%$ reductions in the vibration objective, respectively. The microflap deflection histories corresponding to the various approaches are shown in Fig. 12 for one complete rotor revolution. The flap deflection histories obtained from the SC, AW, and OPT approaches show similarities in the azimuthal locations of the peaks and troughs.

The 4/rev vibratory hub loads obtained using the various saturation approaches for the dual microflap configuration are compared in Fig. 13. The TR and SC approaches yield marginal performance with $13 \%$ and $11 \%$ reductions in the vibration objective, respectively. The AW approach reduces the vibration objective by $88 \%$ whereas the OPT approach yields the best performance with $97 \%$ reduction in the vibration objective. The inboard and outboard microflap deflection histories corresponding to the various saturation approaches are shown in Fig. 14. As in the case of the plain flaps, the AW approach under-utilizes the inboard control surface whereas the OPT approach utilizes both the control surfaces to the fullest possible extent resulting in a significantly better vibration reduction performance.

The different saturation approaches are also compared in terms of their BVI noise reduction capabilities using the dual microflap configuration. Noise levels computed on the carpet plane (depicted in Fig. 4) during active noise reduction using the different saturation approaches are compared to the baseline noise levels in 


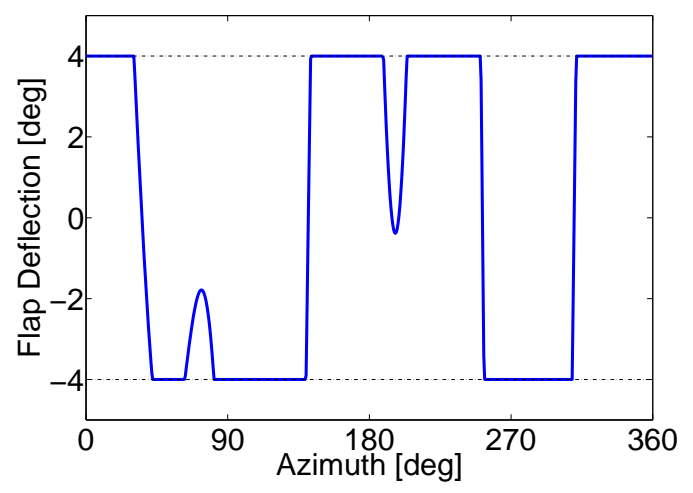

(a) Truncation

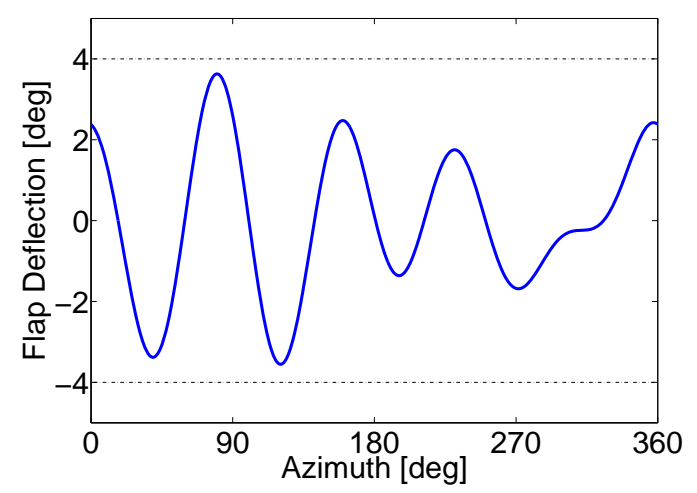

(c) Auto-weighting

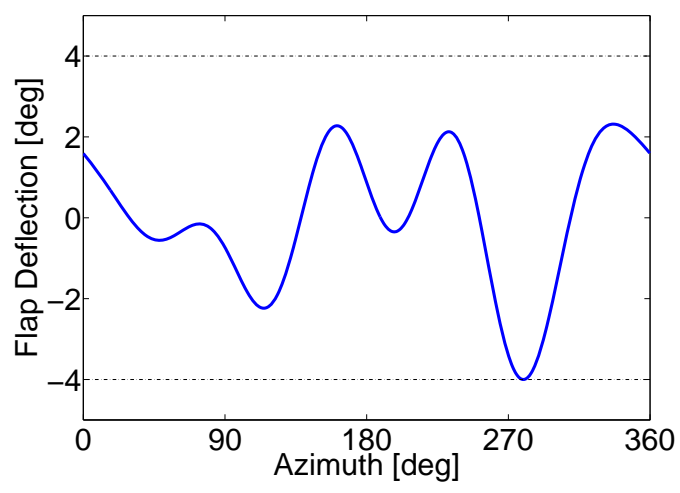

(b) Scaling

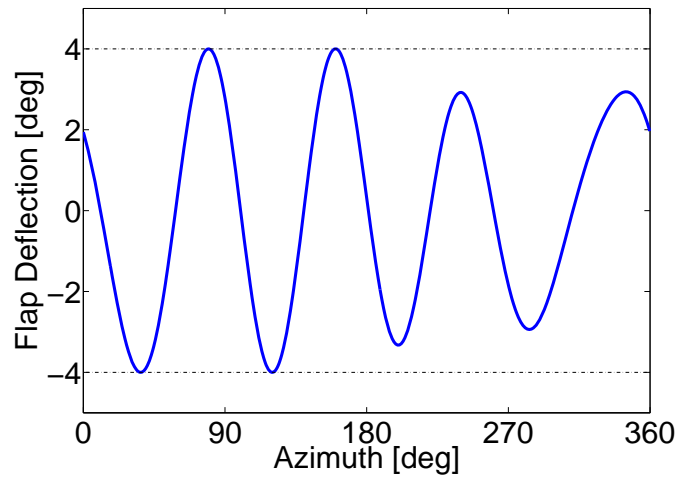

(d) Optimization

Figure 8. Single plain flap deflection histories corresponding to the various saturation approaches at a heavy BVI descending flight condition with $\mu=0.15$.

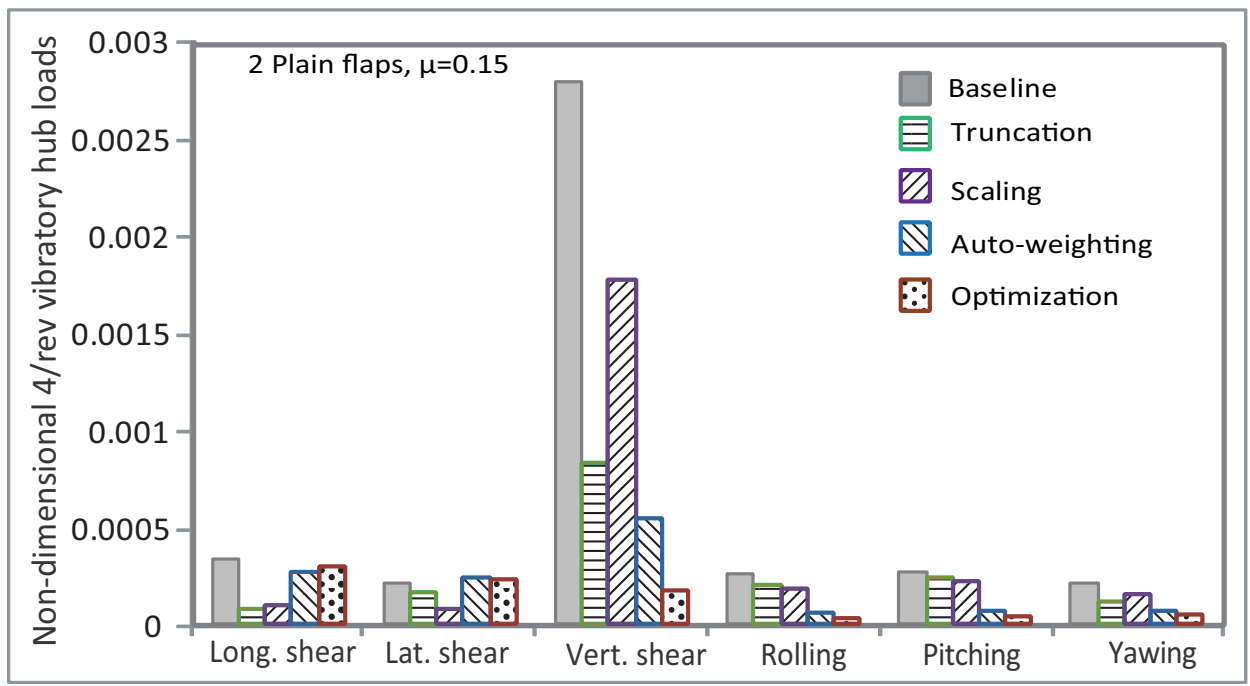

Figure 9. Reduction in 4/rev vibratory hub shears and moments obtained using the various saturation approaches for the dual plain flap configuration at a heavy BVI descending flight condition with $\mu=0.15$. 


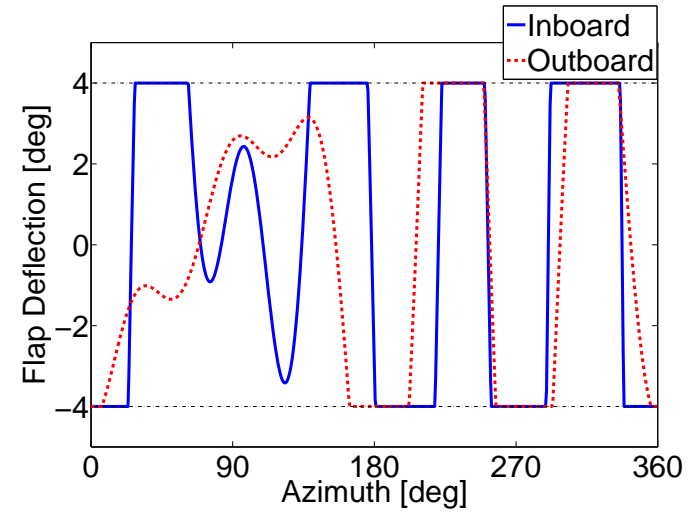

(a) Truncation

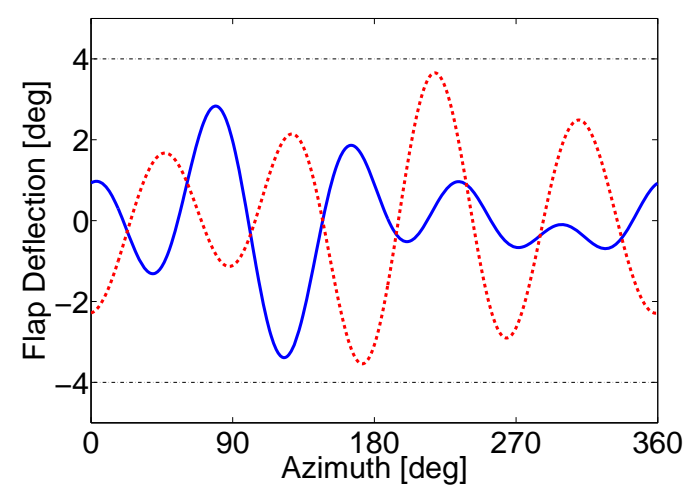

(c) Auto-weighting

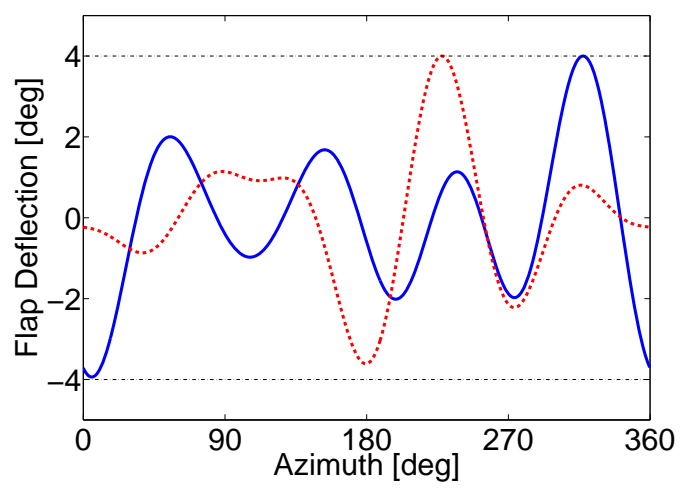

(b) Scaling

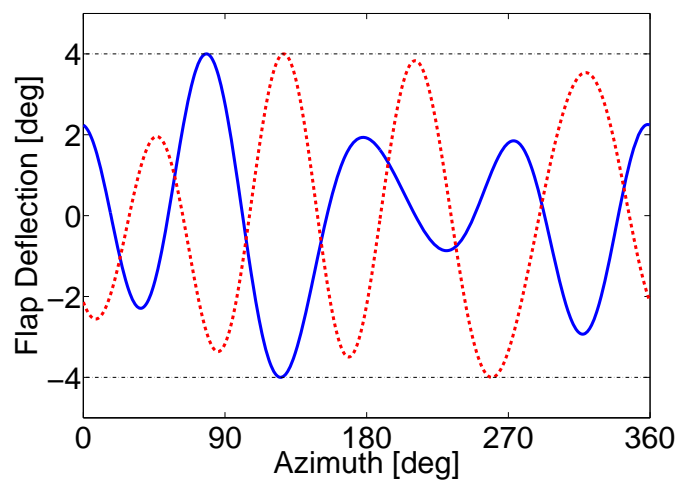

(d) Optimization

Figure 10. Dual plain flap deflection histories corresponding to the various saturation approaches at a heavy BVI descending flight condition with $\mu=0.15$.

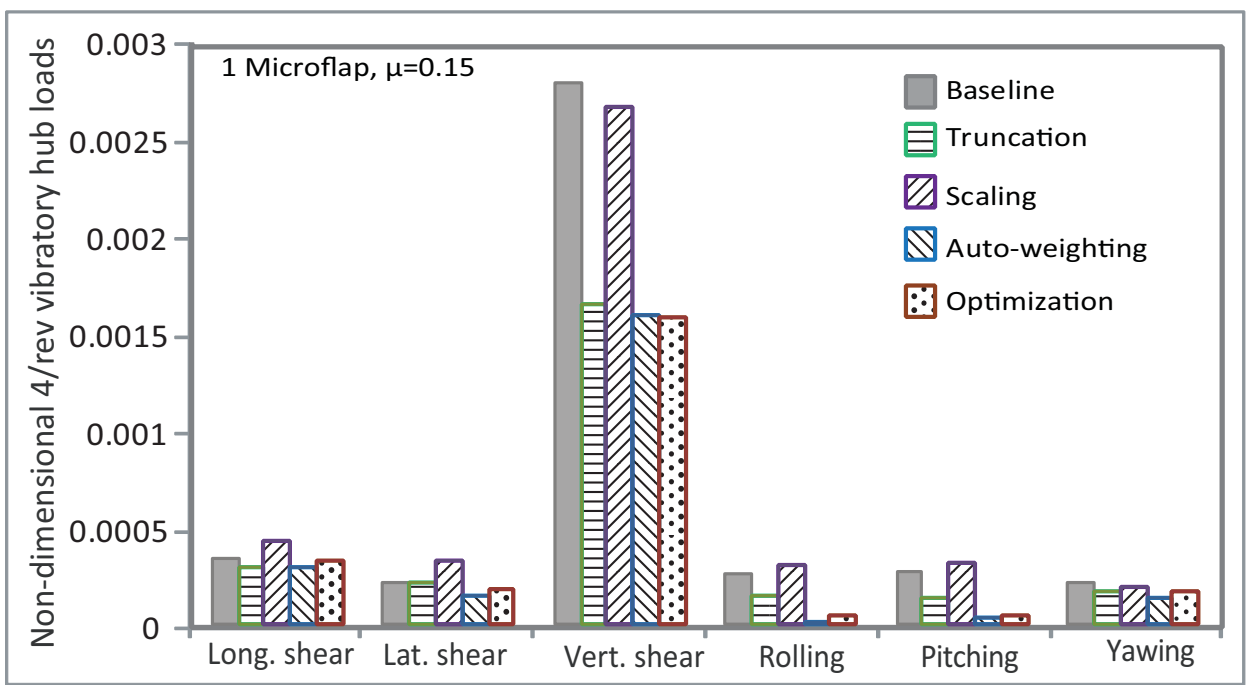

Figure 11. Reduction in 4 /rev vibratory hub shears and moments obtained using the various saturation approaches for the single microflap configuration at a heavy BVI descending flight condition with $\mu=0.15$. 


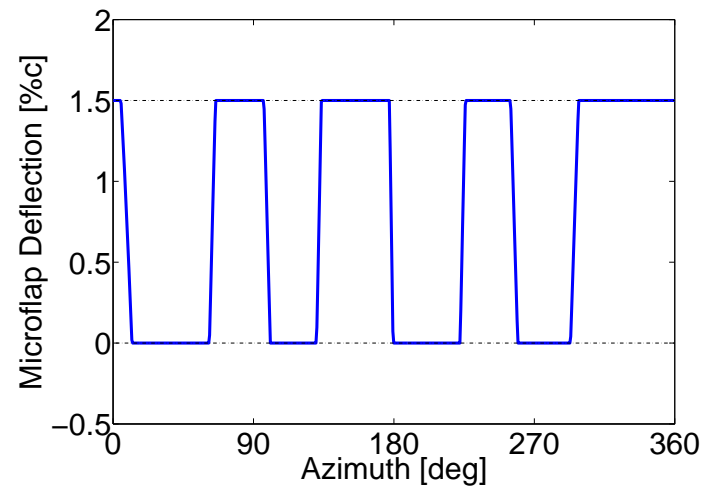

(a) Truncation

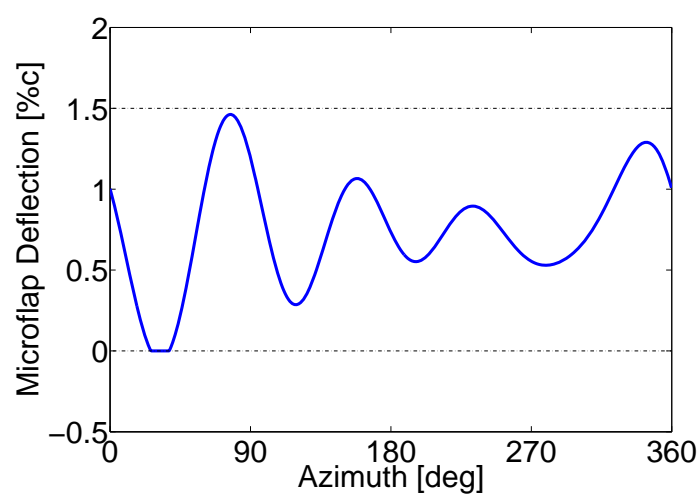

(c) Auto-weighting

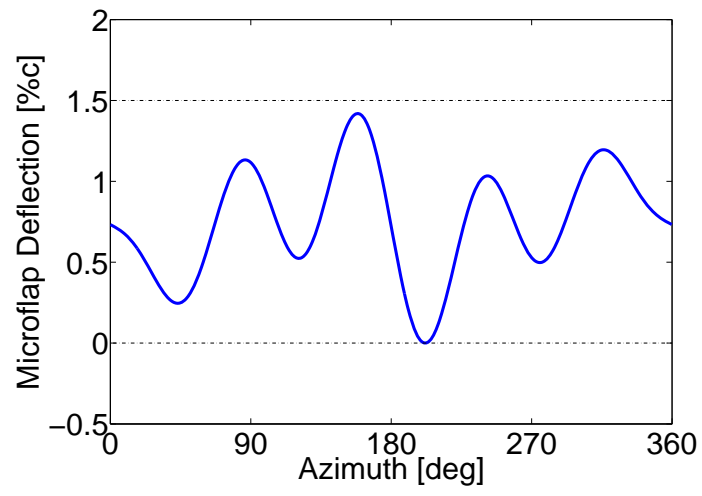

(b) Scaling

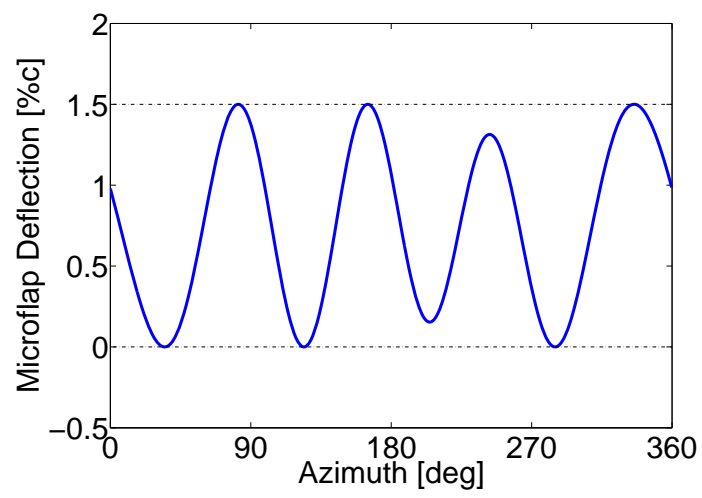

(d) Optimization

Figure 12. Single microflap deflection histories corresponding to the various saturation approaches at a heavy BVI descending flight condition with $\mu=0.15$.

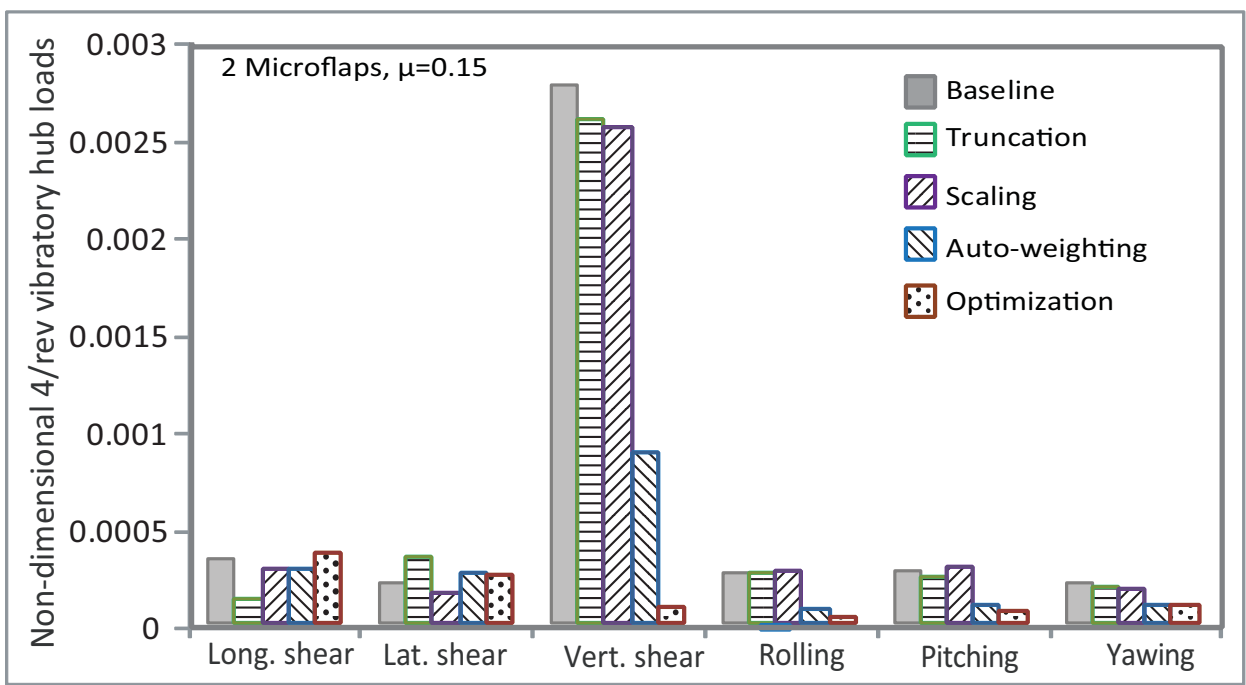

Figure 13. Reduction in 4 /rev vibratory hub shears and moments obtained using the various saturation approaches for the dual microflap configuration at a heavy BVI descending flight condition with $\mu=0.15$. 


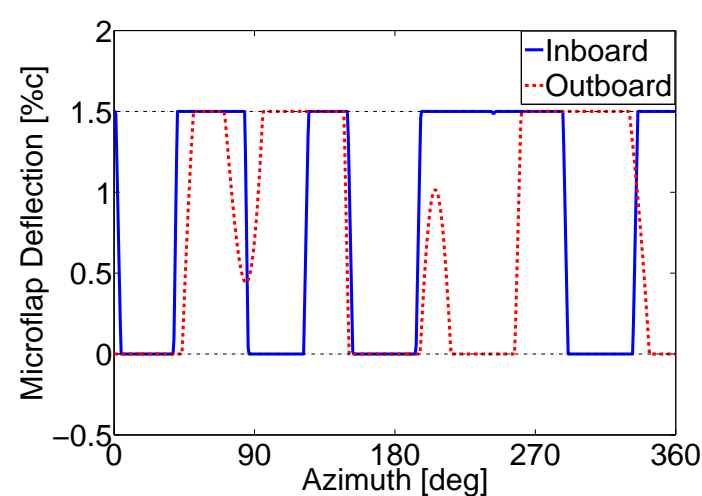

(a) Truncation

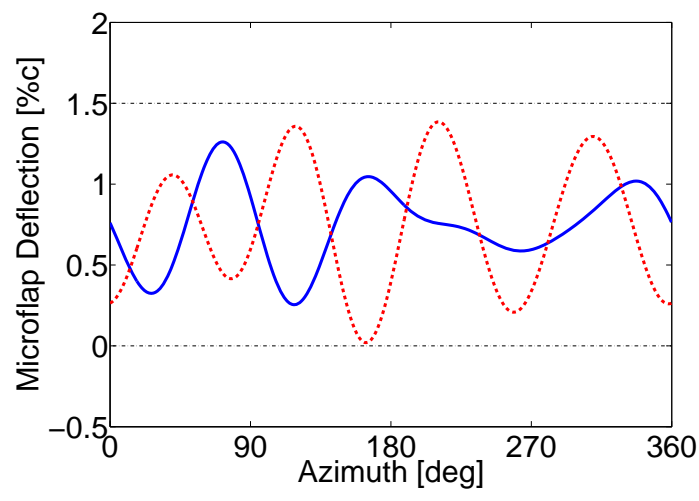

(c) Auto-weighting

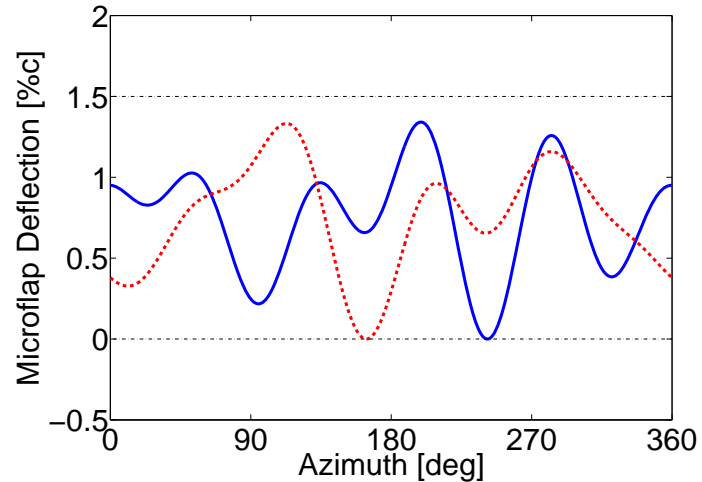

(b) Scaling

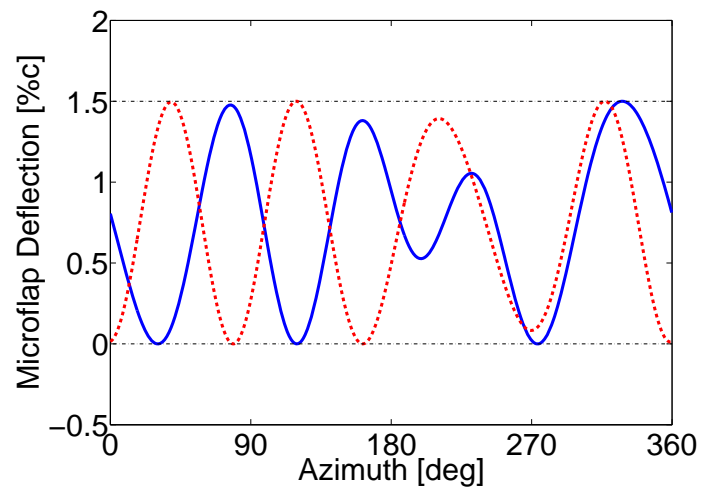

(d) Optimization

Figure 14. Dual microflap deflection histories corresponding to the various saturation approaches at a heavy BVI descending flight condition with $\mu=0.15$. 
Fig. 15. The TR and SC approaches yield similar performance with approximately $1 \mathrm{~dB}$ noise reduction on the advancing side and up to $2 \mathrm{~dB}$ reduction on the retreating side of the rotor disk. By contrast, the AW and OPT approaches yield significantly better performance with a 4-5 dB noise reduction on the advancing side and a 3-4 $\mathrm{dB}$ reduction on the retreating side of the rotor disk. The inboard and outboard microflap deflection histories corresponding to the various saturation approaches are shown in Fig. 16.
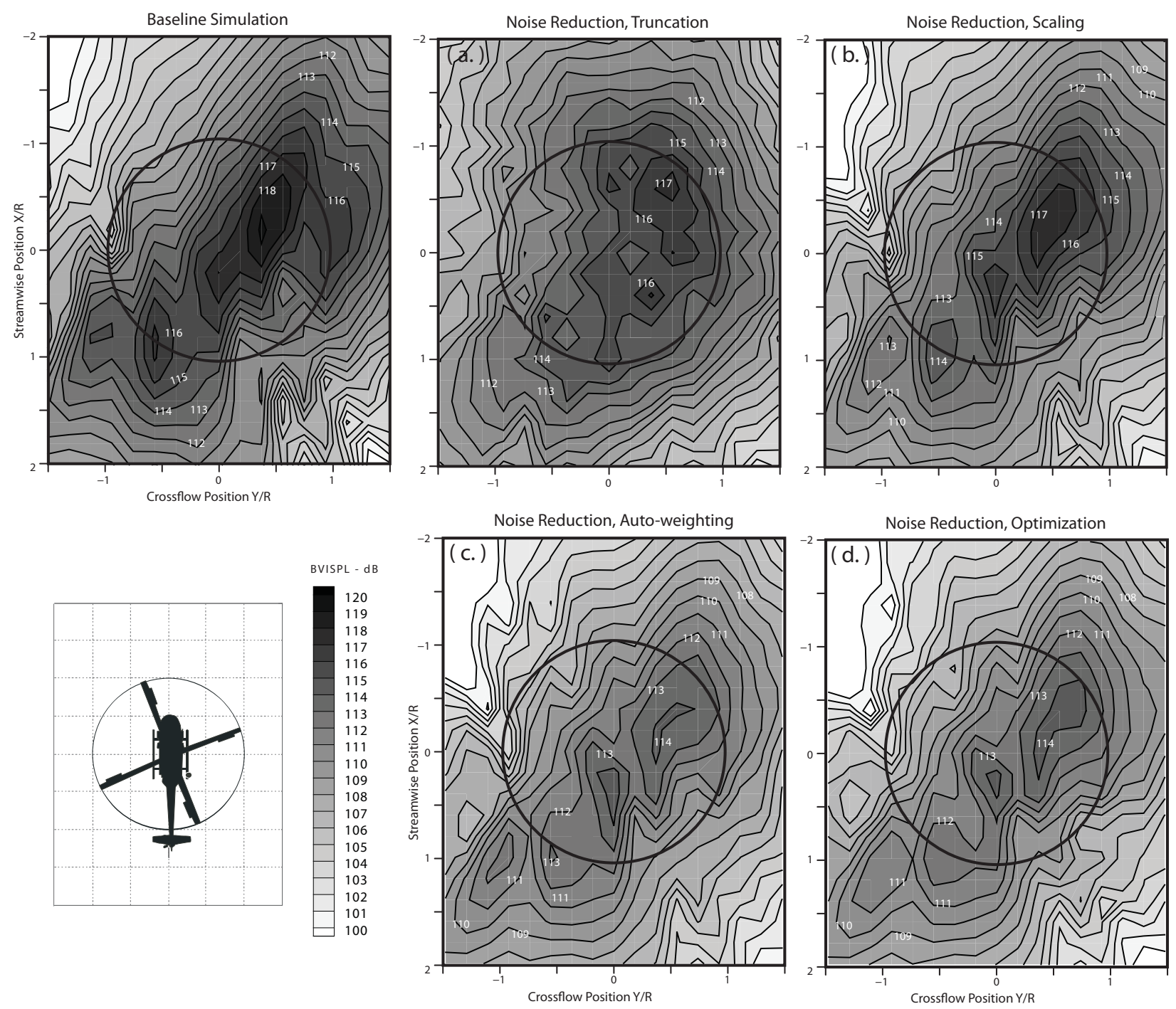

Figure 15. Reduction in noise levels obtained using the various saturation approaches for the dual microflap configuration at a heavy BVI descending flight condition with $\mu=0.15$.

The different saturation approaches are also compared in terms of their simultaneous BVI noise and vibration reduction capabilities using the dual microflap configuration. For the simulations performed using the dual microflap configuration, the relative weighting factor $W_{\alpha}=0.6$ is chosen as it yields an optimal reduction in the vertical hub shear and the feedback microphone noise levels. The noise levels computed on the carpet plane during simultaneous reduction using the different saturation approaches are compared to the baseline noise levels in Fig. 17. The TR approach reduces the noise levels by $1 \mathrm{~dB}$ on both the advancing and retreating sides of the rotor disk. The SC, AW, and the OPT approaches yield similar performance with $2 \mathrm{~dB}$ noise reduction on both the advancing and retreating sides. The $4 /$ rev vibratory hub loads obtained during simultaneous reduction using the various saturation approaches are compared to the baseline levels in Fig. 18. The TR and SC approaches result in a $29 \%$ and $9 \%$ increase in the vibration objective, respectively. The AW approach yields $23 \%$ reduction whereas the OPT approach yields a $29 \%$ reduction in the vibration objective. Significantly better performance obtained using the AW and OPT approaches is apparent in the vertical hub shear component. The TR and SC approaches reduce the vertical 


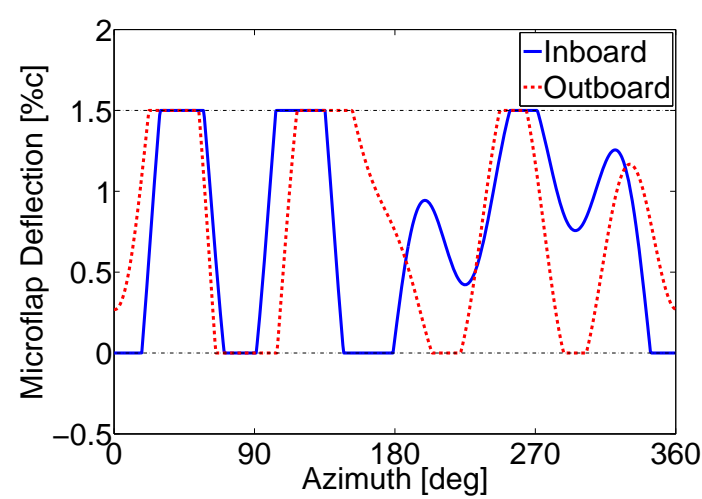

(a) Truncation

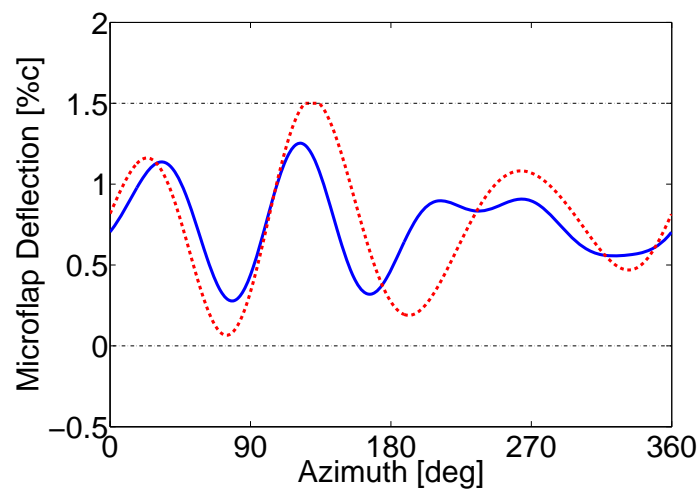

(c) Auto-weighting

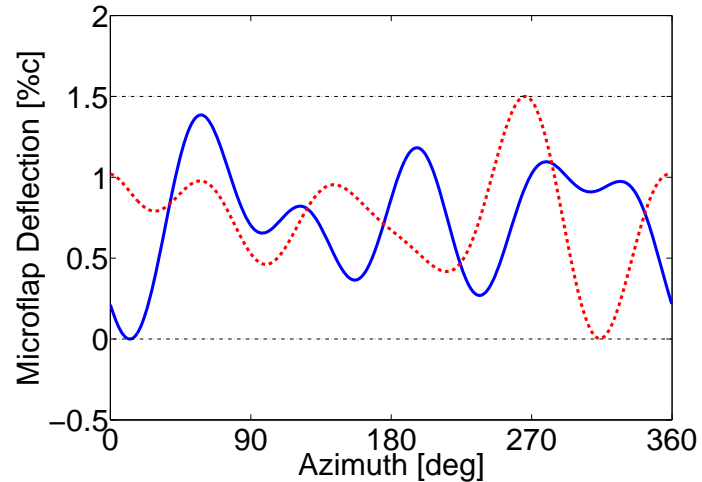

(b) Scaling

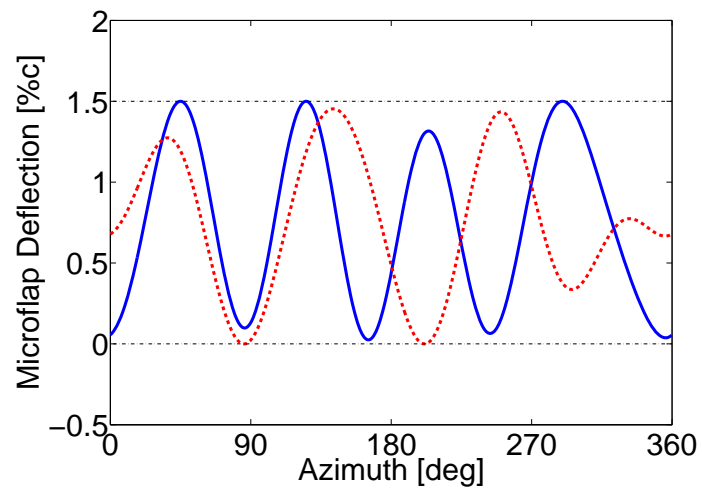

(d) Optimization

Figure 16. Dual microflap deflection histories corresponding to the various saturation approaches during active noise reduction at a heavy BVI descending flight condition with $\mu=0.15$. 
hub shear by $3 \%$ and $10 \%$, respectively, whereas, the AW and OPT approaches reduce the vertical hub shear by $34 \%$ and $37 \%$, respectively. Overall, the AW and OPT approaches yield similar simultaneous BVI noise and vibration reduction performance. However, the OPT approach requires significantly less computational time to converge taking only 10 control updates ( 80 rotor revolutions) compared to over 100 control updates (800 rotor revolutions) taken by the AW approach. The inboard and outboard microflap deflection histories corresponding to the various saturation approaches are shown in Fig. 19. In the AW approach, the maximum deflection of the inboard microflap is less than the saturation limit.
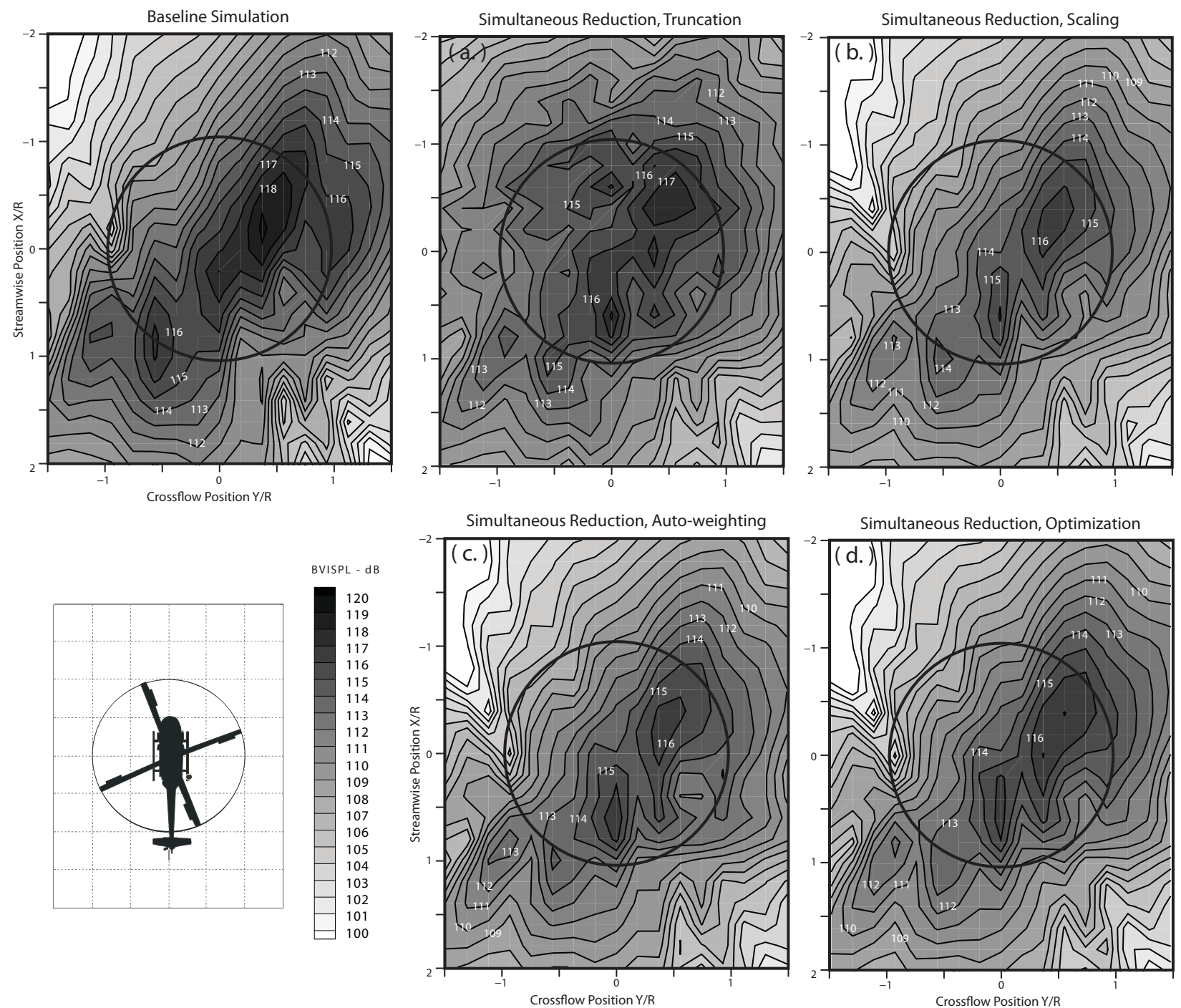

Figure 17. Reduction in noise levels obtained during simultaneous BVI noise and vibration reduction using the various saturation approaches for the dual microflap configuration at a heavy BVI descending flight condition with $\mu=0.15$.

\section{V.B. High-speed Results}

In this section, the vibration reduction performance of the various saturation approaches is compared at a high speed level flight condition with $\mu=0.3$. The $4 /$ rev vibratory hub loads obtained using the various saturation approaches for the single plain flap configuration are compared to the baseline levels in Fig. 20. The TR and SC approaches yield $82 \%$ and $71 \%$ reduction in the vibration objective, respectively. Comparatively, the AW and OPT approaches yield significantly better performance reducing the vibration objective by $93 \%$ and $97 \%$, respectively. The deflection time histories corresponding to the various saturation approaches over one rotor revolution are shown in Fig. 21. A relatively good performance obtained from the TR approach can be attributed to the fact that only a small portion of the flap deflection shown in Fig. 21(a) is being 


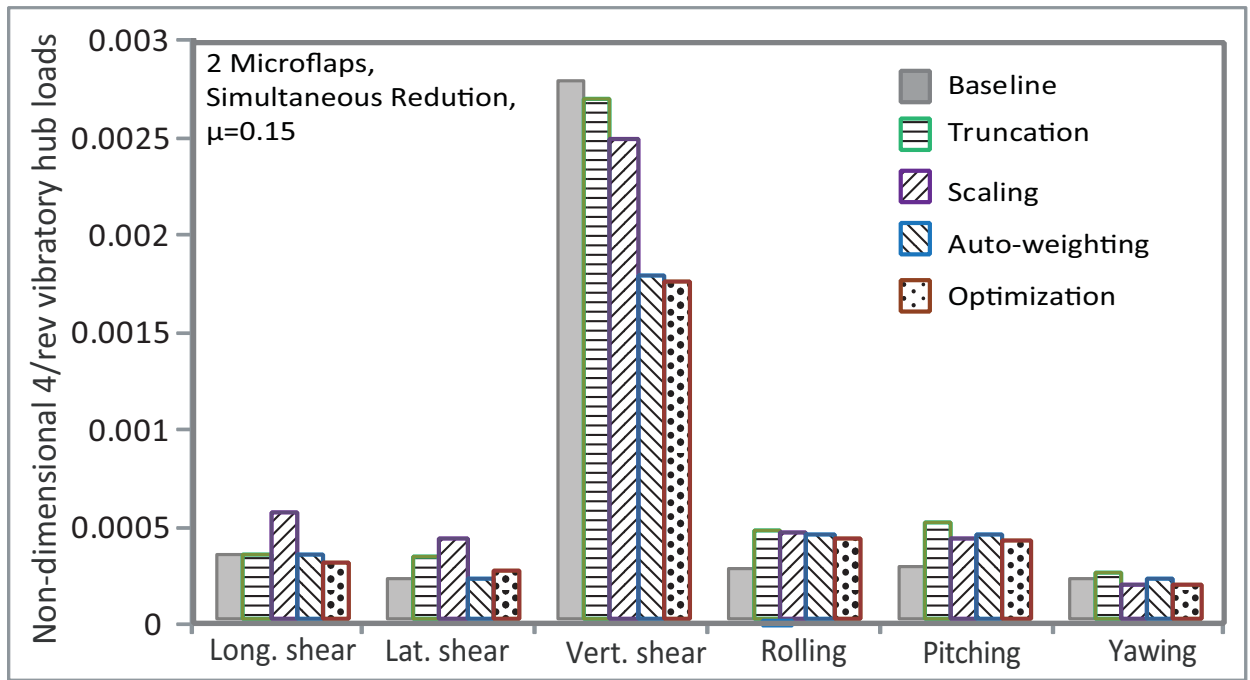

Figure 18. Reduction in 4 /rev vibratory hub shears and moments obtained during simultaneous BVI noise and vibration reduction using the various saturation approaches for the dual microflap configuration at a heavy $B V I$ descending flight condition with $\mu=0.15$.

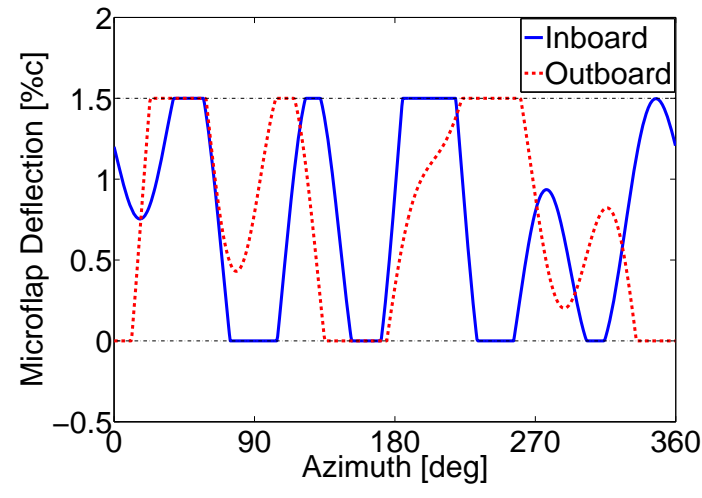

(a) Truncation

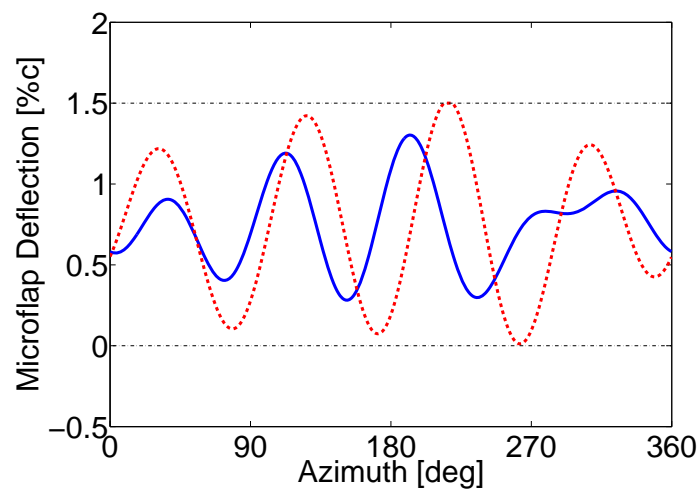

(c) Auto-weighting

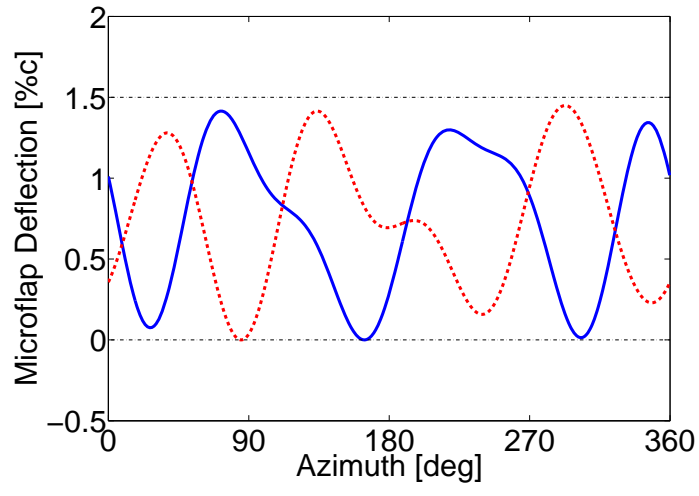

(b) Scaling

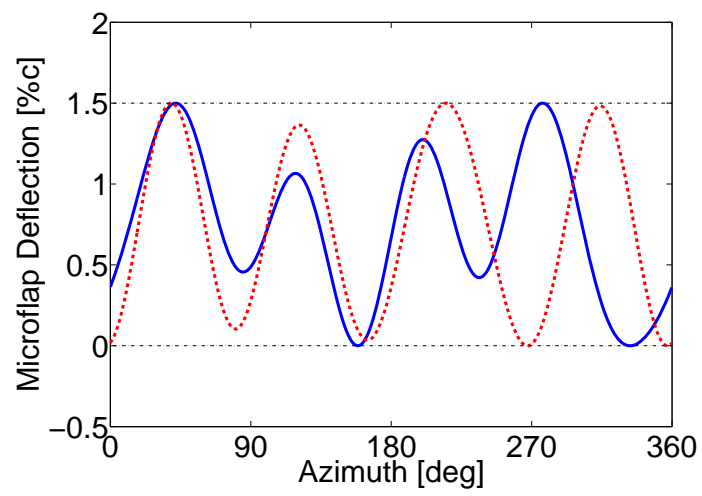

(d) Optimization

Figure 19. Dual microflap deflection histories corresponding to the various saturation approaches at a heavy BVI descending flight condition with $\mu=0.15$. 
truncated. The flap deflection histories corresponding to the SC, AW, and OPT approaches show a qualitative resemblance with similar azimuthal locations for the peaks and troughs.

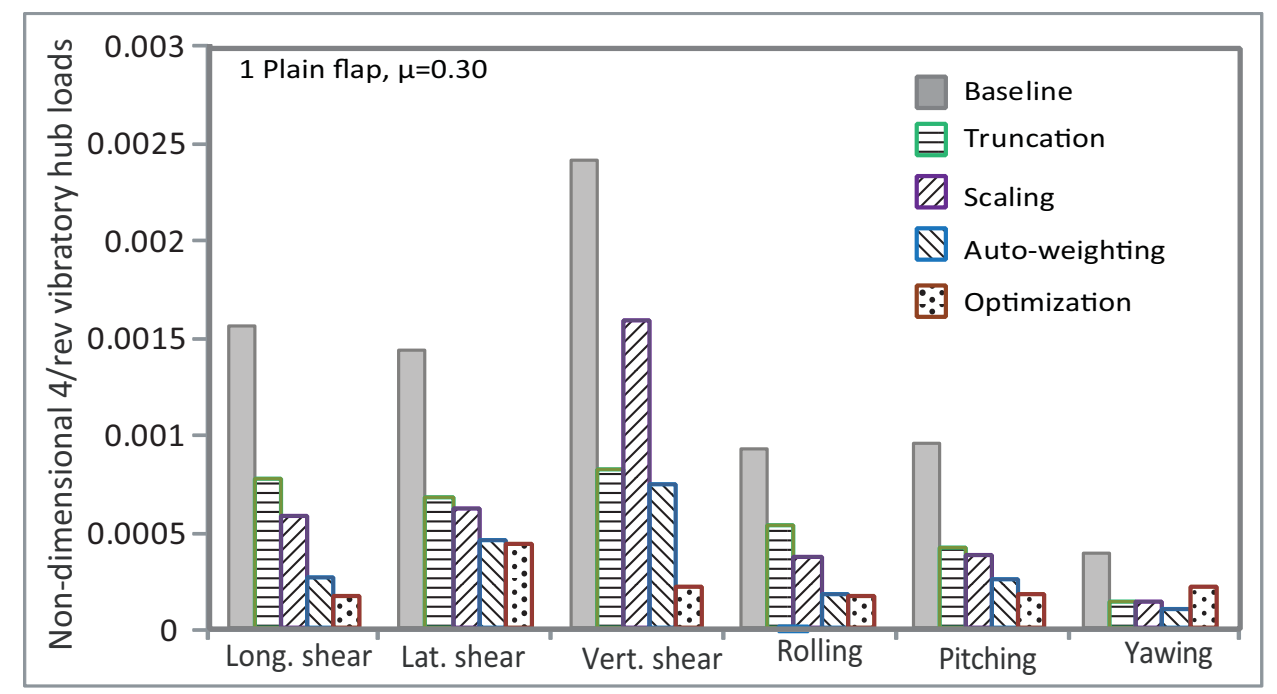

Figure 20. Reduction in 4 /rev vibratory hub shears and moments obtained using the various saturation approaches for the single plain flap configuration at a high-speed flight condition with $\mu=0.3$.

Vibratory hub loads obtained using the various saturation approaches for the dual plain flap configuration are shown in Fig. 22. The TR approach causes an increase in the longitudinal and lateral shears and yields no significant reduction in the vibration objective. The SC approach reduces the vibration objective by $62 \%$ whereas the AW and the OPT approaches yield exceptional performance with $95 \%$ and $99 \%$ reductions in the vibration objective, respectively. The inboard and outboard flap deflection time histories corresponding to the various approaches are shown in Fig. 23. In the TR approach, significant portions of the inboard and outboard flap deflections are truncated, as shown in Fig. 23(a), resulting in its poor performance. The outboard flap is significantly under-utilized by the AW approach whereas the OPT approach utilizes both the flaps to the maximum possible extent.

Similar comparisons are also performed using single and dual microflap configurations. The 4/rev vibratory hub loads for the various saturation approaches are compared in Fig. 24 for the single microflap configuration. The TR approach reduces the vibration objective by $38 \%$. The SC approach causes a significant increase in the vertical hub shear resulting in a $5 \%$ increase in the vibration objective. The AW and OPT approaches yield $91 \%$ and $94 \%$ reductions in the vibration objective, respectively. The microflap deflection time histories corresponding to the various saturation approaches are shown in Fig. 25 for one complete rotor revolution. The microflap deflection histories corresponding to the AW and OPT approaches exhibit similarity in the overall shape.

Vibratory hub loads obtained from the different saturation approaches for the dual microflap configuration are shown in Fig. 26. The TR and SC approaches yield 25\% and 28\% reductions in the vibration objective, respectively. However, both of them cause a small increase in the vertical hub shear. The AW and the OPT approaches yield exceptional performance with $94 \%$ and $98 \%$ reductions in the vibration objective, respectively. The microflap deflection histories corresponding to the various saturation approaches are shown in Fig. 27. The AW approach significantly under-utilizes the outboard microflap whereas the OPT approach utilizes both microflaps to the maximum possible extent.

\section{Summary and Conclusions}

The effect of actuator saturation on the active vibration and noise reduction performance of the conventional trailing-edge plain flaps and the microflaps is examined. A new approach to handling actuator saturation in the HHC algorithm based on constrained nonlinear optimization techniques is developed. Vibration reduction performance of this approach is compared to the existing approaches at various flight conditions. Noise reduction and simultaneous vibration and noise reduction performance of the various 


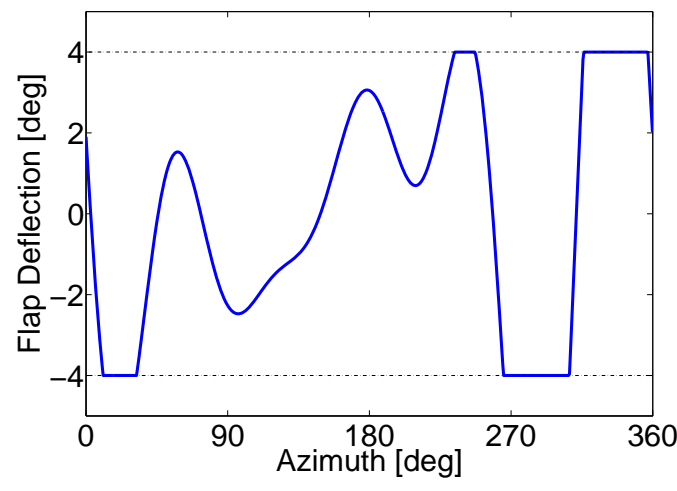

(a) Truncation

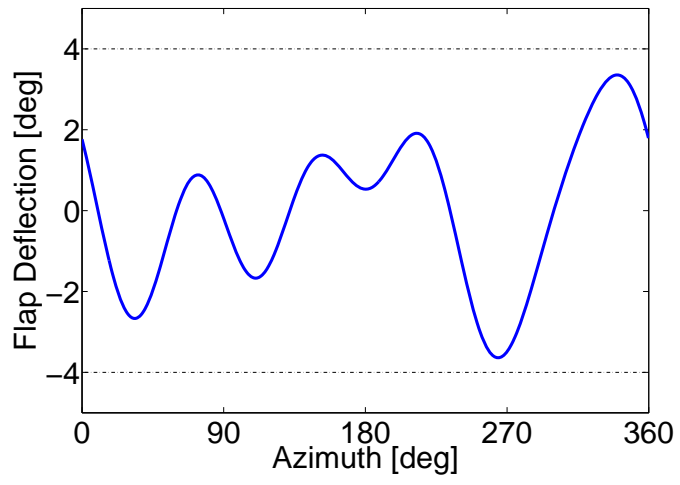

(c) Auto-weighting

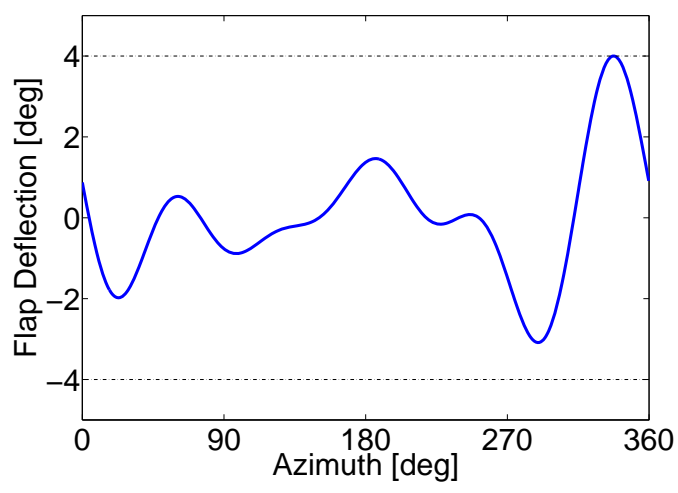

(b) Scaling

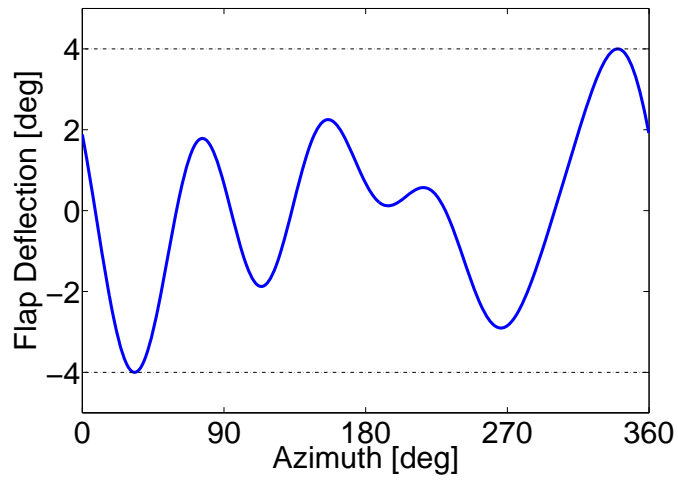

(d) Optimization

Figure 21. Single plain flap deflection histories corresponding to the various saturation approaches at a high-speed flight condition with $\mu=0.3$.

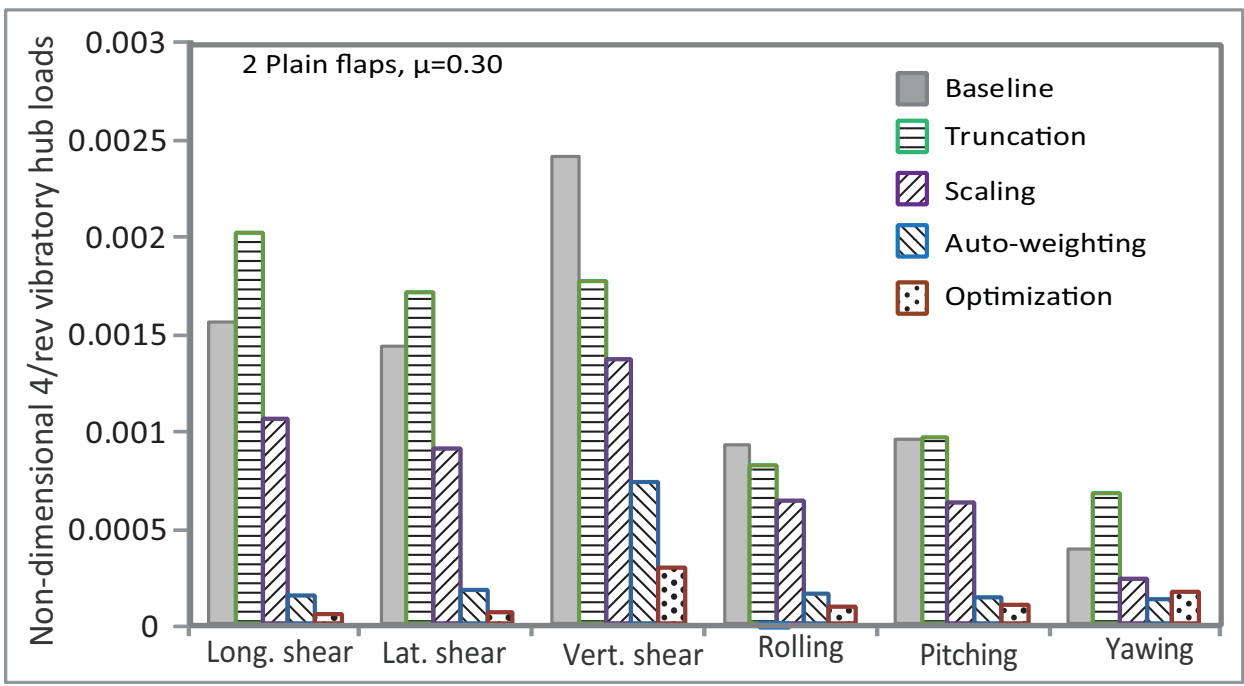

Figure 22. Reduction in 4 /rev vibratory hub shears and moments obtained using the various saturation approaches for the dual plain flap configuration at a high-speed flight condition with $\mu=0.3$. 


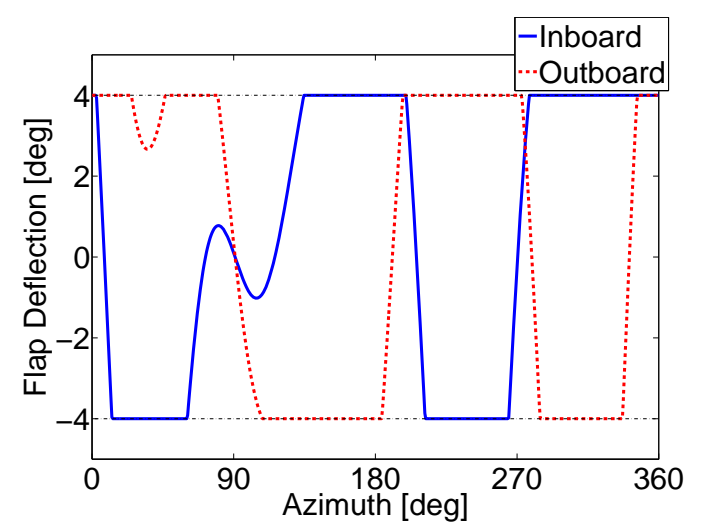

(a) Truncation

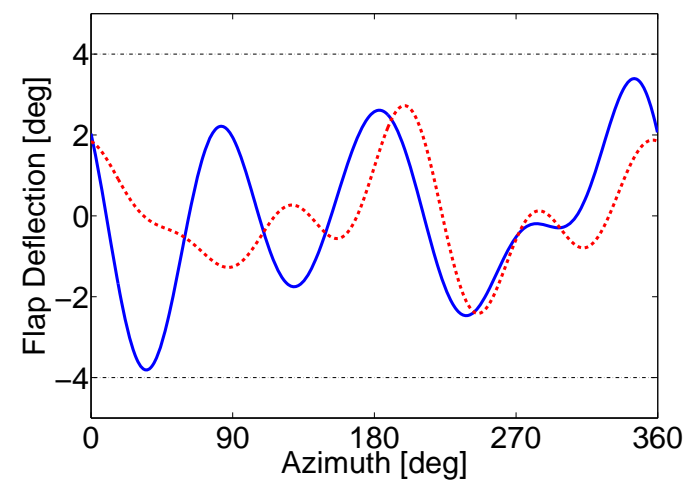

(c) Auto-weighting

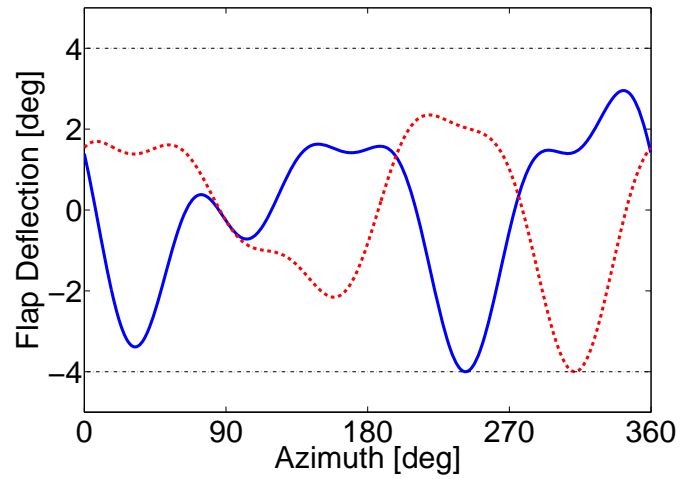

(b) Scaling

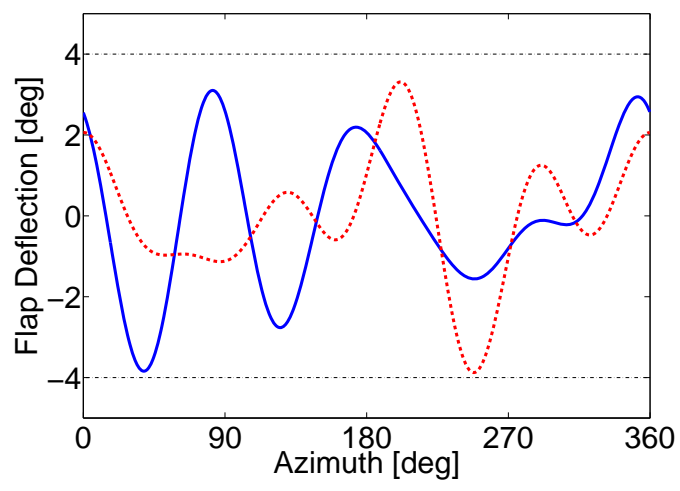

(d) Optimization

Figure 23. Dual plain flap deflection histories corresponding to the various saturation approaches at a high-speed flight condition with $\mu=0.3$.

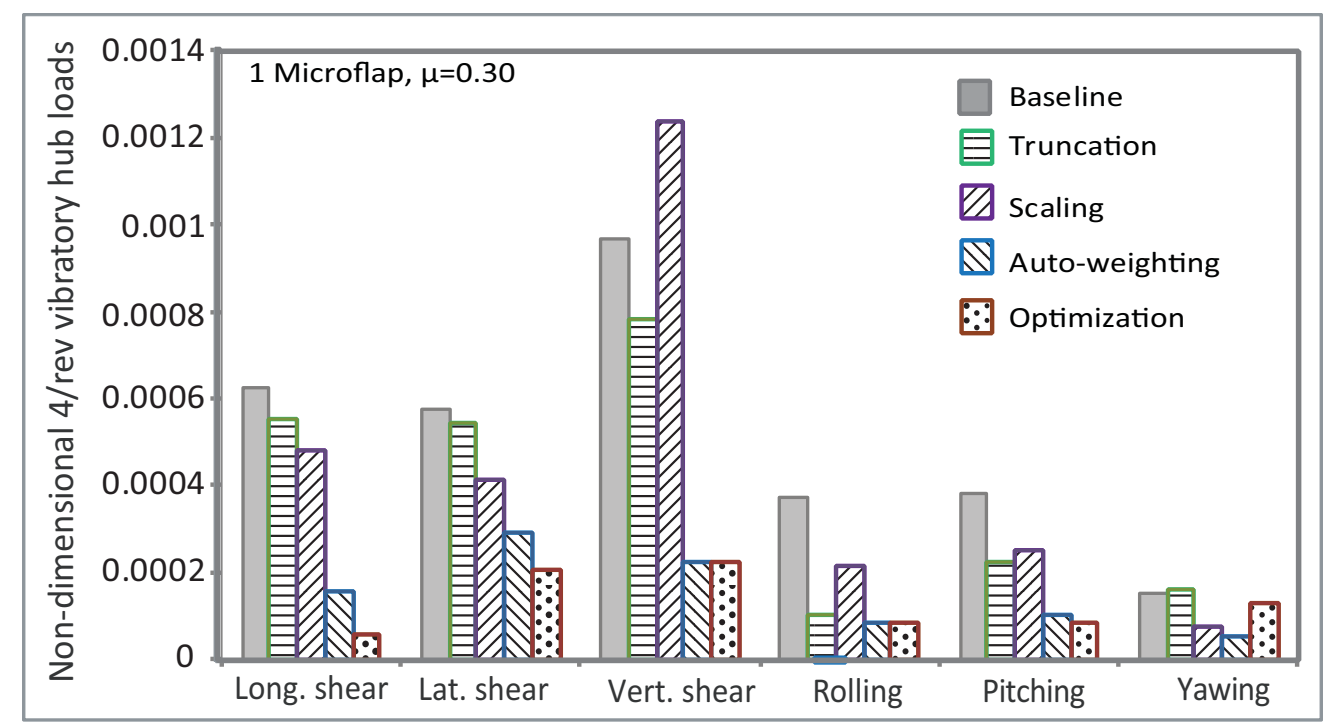

Figure 24. Reduction in $4 /$ rev vibratory hub shears and moments obtained using the various saturation approaches for the single microflap configuration at a high-speed flight condition with $\mu=0.3$. 


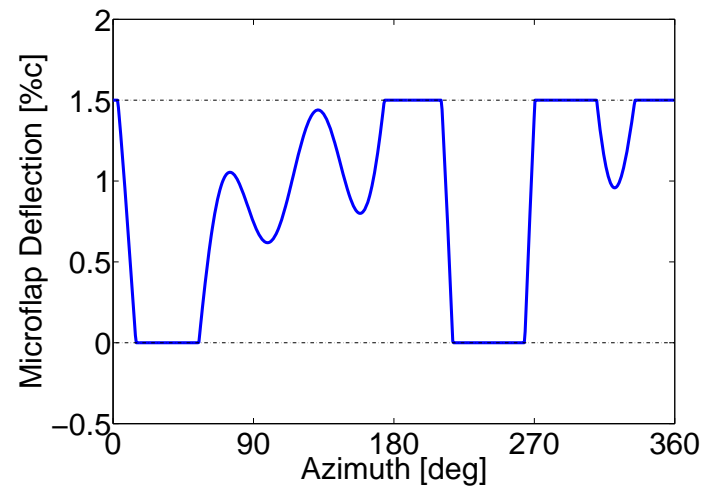

(a) Truncation

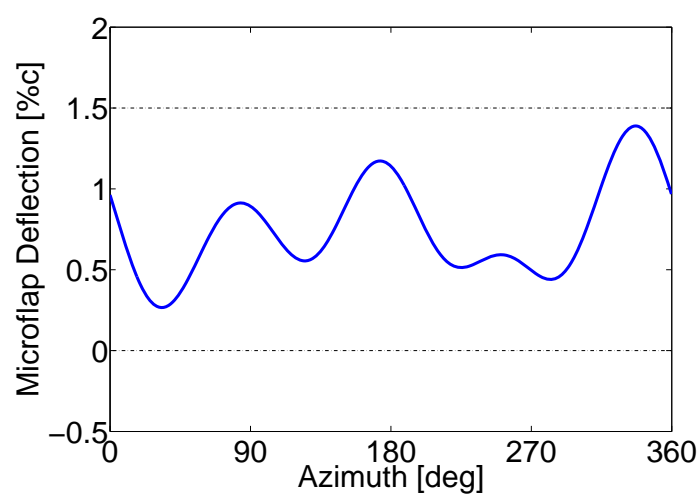

(c) Auto-weighting

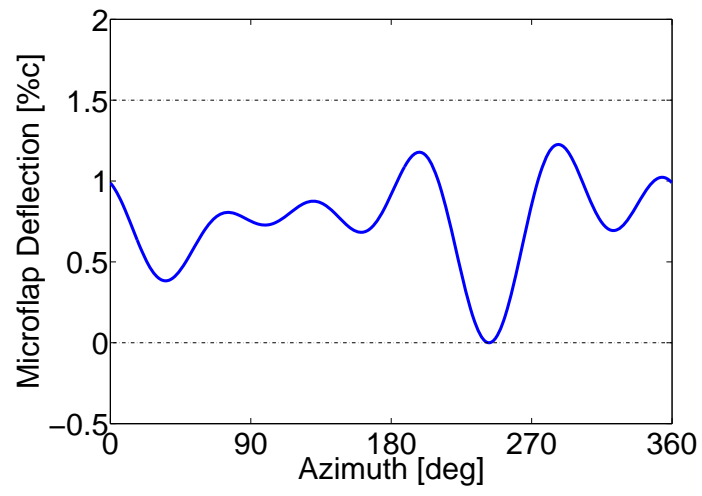

(b) Scaling

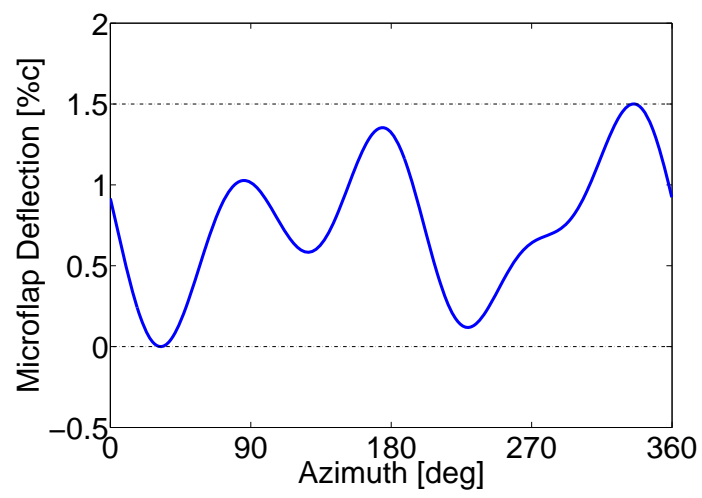

(d) Optimization

Figure 25. Single microflap deflection histories corresponding to the various saturation approaches at a high-speed flight condition with $\mu=0.3$.

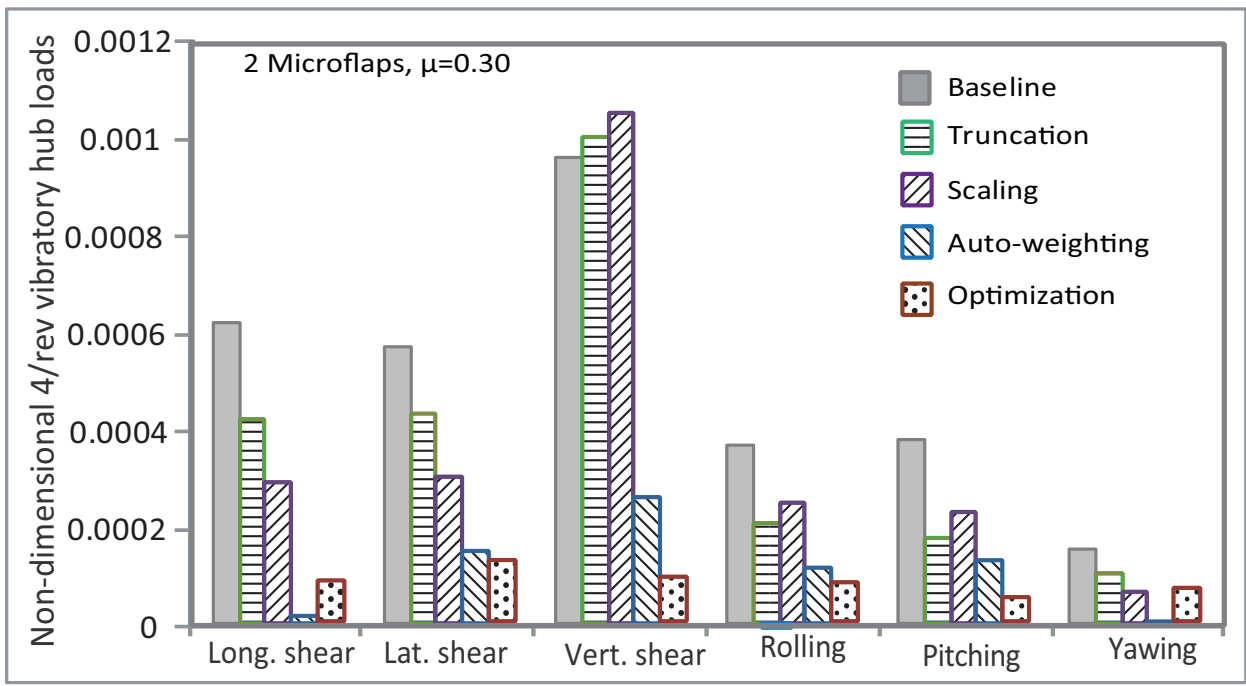

Figure 26. Reduction in 4 /rev vibratory hub shears and moments obtained using the various saturation approaches for the dual microflap configuration at a high-speed flight condition with $\mu=0.3$. 


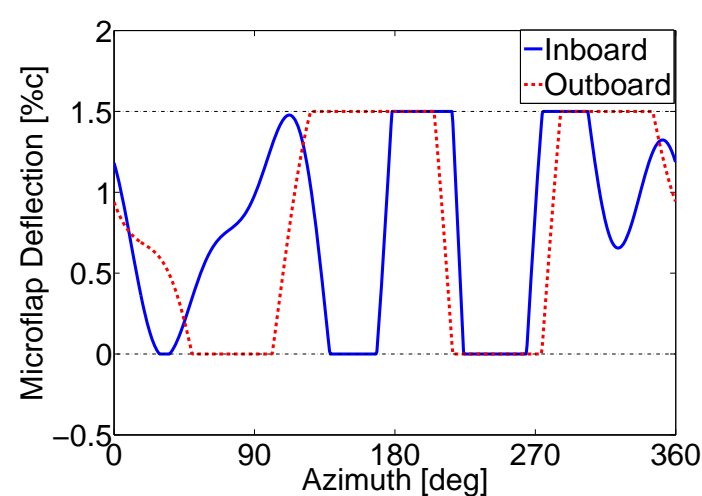

(a) Truncation

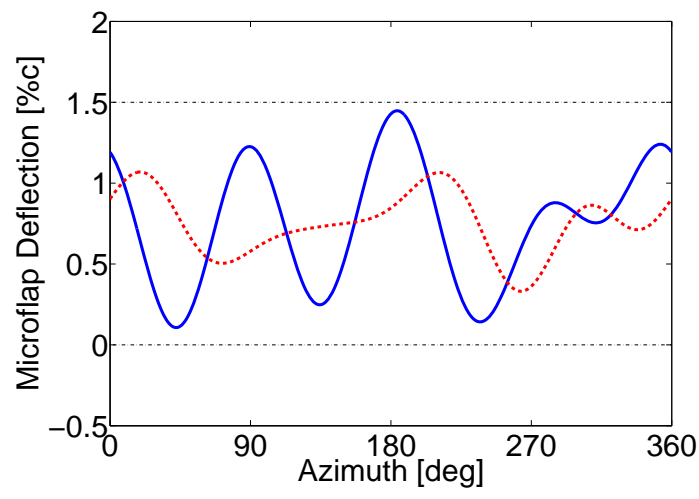

(c) Auto-weighting

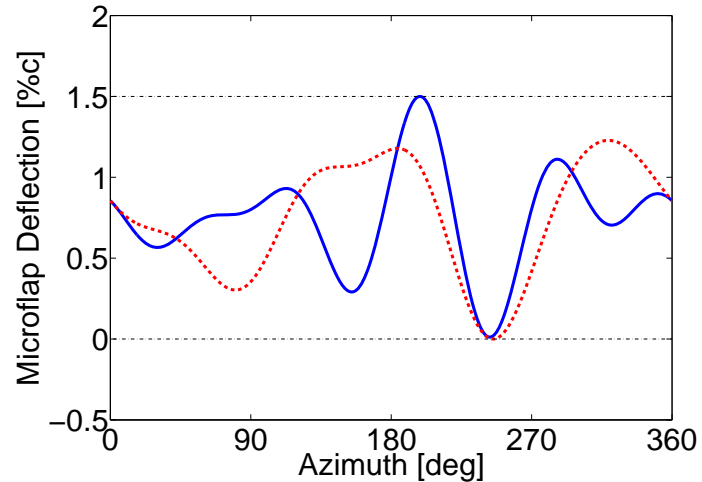

(b) Scaling

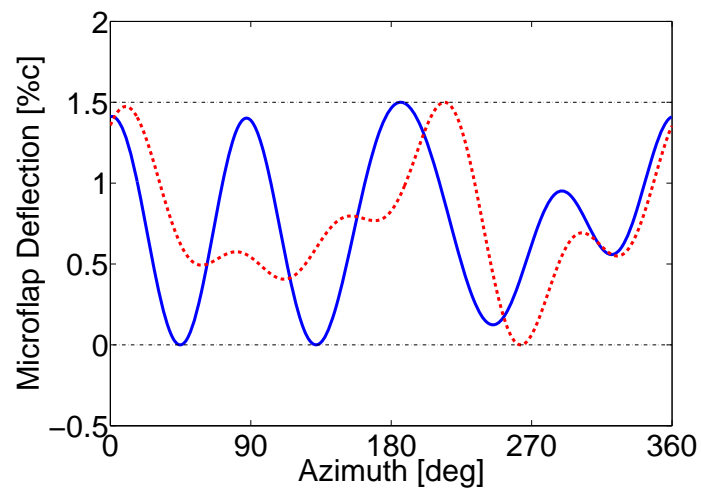

(d) Optimization

Figure 27. Dual microflap deflection histories corresponding to the various saturation approaches at a high-speed flight condition with $\mu=0.3$. 
approaches is compared at a heavy BVI low-speed descending flight condition. The principal findings and conclusions of the study are summarized below.

1. The truncation and scaling approaches are inconsistent and yield only limited vibration or noise reduction using plain trailing-edge flaps or microflaps. By comparison, the auto-weighting and optimization approaches show very good performance.

2. In the case of multiple control surfaces, the optimization approach utilizes all of them to the maximum possible extent producing significantly better vibration reduction performance. In the auto-weighting approach, the weighting on the control inputs corresponding to the various control surfaces is identical. This results in an optimal control input only for one of the control surfaces leaving the others underutilized. In the optimization approach, the deflections corresponding to the various control surfaces are optimized independently and hence are utilized to the maximum possible extent resulting in a better vibration reduction performance.

3. The optimization approach takes significantly less computational time compared to the auto-weighting approach. This is primarily because the auto-weighting approach relies on an a priori guess of the upper bound of the optimal control weighting and involves iteratively adjusting the weighting matrix $\mathbf{R}$ until a control input satisfying the saturation constraints is obtained.

Thus the optimization based approach is the best option for handling actuator saturation constraints in the HHC algorithm during on-blade vibration and noise reduction using flaps and microflaps, especially for the case of multiple control surfaces.

\section{Acknowledgments}

This research was supported by the Vertical Lift Research Center of Excellence (VLRCOE) sponsored by NRTC and U.S. Army with Dr. M. Rutkowski as grant monitor.

\section{References}

${ }^{1}$ Friedmann, P. P. and Millott, T. A., "Vibration Reduction in Rotorcraft Using Active Control: A Comparison of Various Approaches," Journal of Guidance, Control, and Dynamics, Vol. 18, No. 4, July-August 1995, pp. 664-673.

${ }^{2}$ Swanson, S. M., Jacklin, S. A., Blaas, A., Niesl, G., and Kube, R., "Reduction of Helicopter BVI Noise, Vibration, and Power Consumption through Individual Blade Control," Proceedings of the 51st Annual Forum of the American Helicopter Society, Fort Worth, TX, May 1995, pp. 662-680.

${ }^{3}$ Jacklin, S. A., Haber, A., de Simone, G., Norman, T., Kitaplioglu, C., and Shinoda, P., "Full-Scale Wind Tunnel Test of an Individual Blade Control System for a UH-60 Helicopter," Proceedings of the 58th Annual Forum of the American Helicopter Society, Montreal, Canada, June 2002.

${ }^{4}$ Millott, T. A. and Friedmann, P. P., Vibration Reduction in Helicopter Rotors Using an Actively Controlled Partial Span Trailing Edge Flap Located on the Blade, NASA CR 4611, June 1994.

${ }^{5}$ Koratkar, N. A. and Chopra, I., "Wind Tunnel Testing of a Smart Rotor Model with Trailing Edge Flaps," Journal of the American Helicopter Society, Vol. 47, No. 4, Oct. 2002, pp. 263-272.

${ }^{6}$ Patt, D., Liu, L., and Friedmann, P. P., "Simultaneous Vibration and Noise Reduction in Rotorcraft Using Aeroelastic Simulation," Journal of the American Helicopter Society, Vol. 51, No. 2, April 2006, pp. 127-140.

${ }^{7}$ Straub, F. K., Anand, V., Birchette, T. S., and Lau, B. H., "Wind Tunnel Test of the SMART Active Flap Rotor," Proceedings of the 65th American Helicopter Society Annual Forum, Grapevine,TX, May 2009.

${ }^{8}$ Shin, S. J., Cesnik, C. E. S., and Hall, S. R., "Closed-Loop Control Tests of the NASA/ARMY/MIT Active Twist Rotor for Vibration Reduction," Proceedings of the American Helicopter Society 59th Annual Forum, Phoenix, AZ, June 2003.

${ }^{9}$ Bernhard, A. and Chopra, I., "Hover Test of Mach-Scale Active Twist Rotor Using Piezo-Bending-Torsion Actuators," Journal of Aircraft, Vol. 39, No. 4, 2002, pp. 678-688.

${ }^{10}$ Liu, L., Padthe, A. K., and Friedmann, P. P., "Computational Study of Microflaps with Application to Vibration Reduction in Helicopter Rotors," AIAA Journal, Vol. 49, No. 7, July 2011, pp. 1450-1465.

${ }^{11}$ Padthe, A. K., Liu, L., and Friedmann, P. P., "Numerical Evaluation of Microflaps for On Blade Control of Noise and Vibration," Proceedings of the 52nd AIAA/ASME/ASCE/AHS/ACS Structures, Structural Dynamics and Materials Conference, Denver, CO, April 2011.

${ }^{12}$ Min, B. Y., Sankar, L., and Bauchau, O. A., "A CFD-CSD Coupled Analysis of Hart II Rotor Vibration Reduction Using Gurney Flaps," Proceedings of the 66th American Helicopter Society Annual Forum, Phoenix, AZ, May 11-13 2010.

${ }^{13}$ Bernstein, D. S. and Michel, A. N., "A Chronological Bibliography on Saturating Actuators," International Journal of Robust and Nonlinear Control, Vol. 5, 1995, pp. 375-380. 
${ }^{14}$ Myrtle, T. F. and Friedmann, P. P., "Application of a New Compressible Time Domain Aerodynamic Model to Vibration Reduction in Helicopters Using an Actively Controlled Flap," Journal of the American Helicopter Society, Vol. 46, No. 1, Jan. 2001, pp. 32-43.

${ }^{15}$ Patt, D., Liu, L., Chandrasekar, J., Bernstein, D. S., and Friedmann, P. P., "Higher-Harmonic-Control Algorithm for Helicopter Vibration Reduction Revisited," Journal of Guidance, Control, and Dynamics, Vol. 28, No. 5, September-October 2005, pp. 918-930.

${ }^{16}$ Johnson, W., Self-Tuning Regulators for Multicyclic Control of Helicopter Vibrations, NASA Technical Paper 1996, 1982.

${ }^{17}$ Cribbs, R. and Friedmann, P. P., "Actuator Saturation and Its influence on Vibration Reduction by Actively Controlled Flaps," AIAA Paper No. 2001-1467. Proceedings of the 42nd AIAA/ASME/ASCE/AHS/ACS Structures, Structural Dynamics and Materials Conference, Seattle, WA, April 2001.

${ }^{18}$ Roget, B. and Chopra, I., "Closed-Loop Test of a Rotor with Individually Controlled Trailing-Edge Flaps for Vibration Reduction," Journal of the American Helicopter Society, Vol. 55, No. 1, 2010.

${ }^{19}$ Glaz, B., Friedmann, P. P., Liu, L., Kumar, D., and Cesnik, C. E. S., "The AVINOR Aeroelastic Simulation Code and Its Application to Reduced Vibration Composite Rotor Blade Design," Proceedings of the 50th AIAA/ASME/ASCE/AHS/ACS Structures, Structural Dynamics and Materials Conference, Palm Springs, CA, May 2009, AIAA Paper No. $2009-2601$.

${ }^{20}$ de Terlizzi, M. and Friedmann, P. P., "Active Control of BVI Induced Vibrations Using a Refined Aerodynamic Model and Experimental Correlation," American Helicopter Society 55th Annual Forum Proceedings, Montreal, Canada, May 25-27 1999, pp. 599-615.

${ }^{21}$ Liu, L., Padthe, A., Friedmann, P. P., Quon, E., and Smith, M., "Unsteady Aerodynamics of an Airfoil/Flap Combination on a Helicopter Rotor Using CFD and Approximate Methods," Journal of American Helicopter Society, Vol. 56, No. 3, July 2011, pp. 1-13.

${ }^{22}$ Brenter, K., A Computer Program Incorporating Realistic Blade Motions and Advanced Acoustic Formulation, NASA Technical Memorandum, Vol. 877211986.

${ }^{23}$ Patt, D., Liu, L., and Friedmann, P. P., "Rotorcraft Vibration Reduction and Noise Prediction Using a Unified Aeroelastic Response Simulation," Journal of the American Helicopter Society, Vol. 50, No. 1, Jan. 2005, pp. 95-106.

${ }^{24}$ Abbott, I. H., Doenhoff, A. E. V., and Stivers, L. S., Summary of Airfoil Data, NACA Report 824, 1945.

${ }^{25}$ Fletcher, R., Practical Methods of Optimization, John Wiley and Sons, 1987.

${ }^{26}$ Schittkowski, K., "NLQPL: A FORTRAN-Subroutine Solving Constrained Nonlinear Programming Problems," Annals of Operations Research, Vol. 5, 1985, pp. 485-500. 